\title{
Vorrede des Herausgeber's zum I. Bande.
}

\section{I. \\ Aus der Jenaer Studienzeit.}

1. Bemerkungen zu Fichtes Grundlage der gesammten Wissenschaftslehre [1794]. S. 3-4.

Die ,Bemerkungen“: verfasste Herbart in dem ersten Semester seiner Studienzeit als Zuhörer in Fichtes Colleg über die Wissenschaftslehre. Nach Hartenstein (SW XII. S. Ix) hat Herbart dieselben Fichte persön lich zur Begutachtung übergeben und Fichtes Urtheil hierüber münd lich empfangen.

Drucke. Die Bemerkungen sind abgedruckt in $\mathrm{K}_{\mathrm{L}} \mathrm{S}_{\mathrm{CH}} \mathrm{l}, \mathrm{xv}-\mathrm{xvI}$ u. SW XII, 3-4.

Grundlage. Der Text vorliegender Ausgabe ist Al,druck des Textes von SW XII, 3-4.

Paginirung. In T'exte ist die Paginirung von sw, unter dem Texte die von Kíścri angegeben.

2. Einige Bumerkungen über den Begriff des Ideals, in Rücksicht auf Rists Aufsatz über moralisehe und aesthetische Ideale ${ }^{1}[1796]$. S. $5-8$.

Rist hatte als auswärtiges Mitglied der literarischen Gesellschaft nach Jena einen Aufsatz über moralische und aesthetische Urtheile gesandt, über welchen besonders Gries ,eine gewaltige Kritik": geübt hatte. Herbart, den an Rist eine innige Freundschaft band und der sich auch „rühmen" durfte, Rist ,besser verstanden zu haben, wie irgend einer" der in Jena verweilenden Freunde, nahm durch die „Bemerkungen über den Begriff des Ideals" Rist in Schutz. Herbart schreibt in dem Briefe rom September 1796: „Ich habe mit Dir nur einen Wortstreit, indessen wünsche ich der Idee über die Production der Ideale, und über die Nothwendigkeit, die Wissenschaftslehre durch die Deduction derselben zu schliessen, Deine Aufmerksamkeit und Prü-

1 Der Aufsatz von Rist folgt als Supllement 3, S. $417-420$. 
fung; denn für die Methode der W.sch.l. und für die Uebersicht derselben scheint sie mir wichtig." Aus diesem Briefe geht mit Evidenz hervor, dass die Abhandlung, welche Hartenstein in das Jahr 1794 verlegt, in das Jahr 1796 gehört. Die Berichtigung des falschen Datums 1794 ist zuerst durch R. Zimmermann erfolgt. S. d. Aufsatz desselben: Perioden in Herbarts philosophischem Geistesgange in den Sitzungsberichten der (Wiener) Akademie der Wissenschaften (philosoph. -histor. Classe) Bd. 83, Jahrg. 1876, S. 179 ff. Die falsche Angabe Hartenstein s beruht übrigens, wie mir derselbe schreibt, auf einem Druckfehler.

Manuscript. In Rists Nachlasse befindet sich ein Theil des Manuscripts (1 Bl. in $\left.4^{\prime}\right)$ rom vorliegenden Aufsatze und zwar der Abschnitt vom Anfang „Einige Bemerkungen über . . . bis S. 6, 24. Hauptideen derselben zu erinnern". Die Fortsetzung, welche Herbart mit den am Schlusse des handschriftlichen Fragmentes stehenden Worten: (,die Fortsetzung nächstens") verspricht und welche Hartenstein wenigstens für die Edition in $\mathrm{KL} S \mathrm{SCH}$ noch rorgelegen hat, scheint verloren gegangen zu sein.

Drucke. Der Aufsatz war bis jetzt nur theilweise abgedruckt und zwar zuerst in $\mathrm{KL}$ ScH I, S. xx-xxIIr u. SW XII, S. 4-7. In $\mathrm{KL} \mathrm{ScH} \mathrm{ist} \mathrm{die} \mathrm{Ueberschrift} \mathrm{richtig} \mathrm{nach} \mathrm{dem} \mathrm{Msc.} \mathrm{angegeben,} \mathrm{während}$ in SW der Titel: „Bruchstück einer Abhandlung aus dem Jahre 1794" (sic) lautet.

Beide Abdrucke beginnen mit den Worten: „Wie sind synthetische Urtheile a priori möglich (S. 6 Z. 25). In $\mathrm{KuSCH}$ leitet Hartenstein den Abdruck mit den Worten ein: Der Aufsatz beginnt mit der Erklärung: ,über ein rhapsodisches Räsonnement lassen sich . . . - verknüpft haben müsste". Eine Vergleichung mit dem Texte der vorliegenden Ausgabe zeigt, dass Hartensteins Angabe nicht richtig ist.

Grundlage. Der rorliegende Text ist rom Anfang bis S. 6 Z. 24 nach dem Manuscript gedruckt, von S. 6 Z. 25 bis zu Ende nach SW XII.

Paginirung. Im Texte vou S. 6 Z. 25 an bis zu Ende ist die Paginirung der Grundlage SW XII angegeben. Unter dem Texte steht die Paginirung von KL SCH I.

\section{Spinoza und Schelling, eine Skizze [1796]. S. $8-11$.}

Dieser Aufsatz ist in der literarischen Gesellschaft im Sommersemester 1796 vorgelesen worden. Herbart schickte ihn an Rist, um dessen Aufmerksamkeit auf Schelling zu lenken.

Herbarts Brief an Rist rom September 1796: „Durch das kleine Blatt über Schelling wünsche ich Deine Aufmerksamkeit auf ihn lenken zu können, wenigstens will ich so den Fehler wieder gut machen, den ich beging, da ich Dir blos das, was er nicht leiste, und sein Missverstehen der W.sch.l. darzustellen suchte." 
Manuscript. Das Msc. (1 Bl. 4", auf beiden Seiten beschrieben) befindet sich in Rists Nachlasse.

Drucke. Der Aufsatz ist abgedruckt in SW XII, 7-10.

Grundlage. Dats Mauuseript.

Textveränderungen. Diese bestehen lediglich in der Ergänzung von Abkürzungen. Das Ergänzte ist in [ j gesetzt worden, sodass die originale Gestaltung des Wortes leicht zu erkennen ist.

Varianten. S. 9. Z. 13. Ysc.: Systeme getreu blieb

Siw: Systeme treu blieb.

S. 10. Z. 1 2. Ysc.: ohne alle übrige

SIV: ohne alle übrigen.

S. 10. Z. 14. Iss.: durch intellectuale SW: durch iniellectuelle.

S. 10. Z. 15. Msse.: sich selbst erzeugt SII: selbst sich erzeugt.

‥ 10. \%. 16. Hsc.: Allmacht SW: Allmacht.

S. 10. Z. 22. Msc.: ist eben durch SW: ist aber durch.

S. 10. Z. 22. Isc.: Entgegensetzung sll: Entgegensetzung.

S. 10. Z. 31. Isc.: Totalität des Seyns unter beyde SW: Totalitit unter beide.

S. 10. Z. 36. Msc.: Dieser letztern SW: Dieser letzten.

S. 11. 'Z. 10 u. 14 [11]. ${ }^{1}$ Mse.: intellectual SW: intellectuell

S. 11. Z. 19-20. Msc.: Idealism u. Realism SW: Idealismus u. Realismus.

Paginirung. Lnter dem Texte ist die entsprechende Paginirung des bisher einzigen Abdruckes in SW XII, 7--10 angegeben worden.

4. Versuch einer Beurtheilung ron Schellings Schrift: Ueber die Möglichkeit einer Form der Philosophie überhaupt [1796]. S. 12-17.

5. Ueber Schellings Sichrift: Vom Ich, oder dem Unbedingten im menschlichen Wissen [1796]. S. 18-36.

Brief Herbarts an smidt: Jena im Anfange December 1796: „Endlich bester Smidt, kann ich Dir den rersprochenen Aufsatz senden. Mein Abschreiber war ein paar Tage krank, und konnte also nicht so

${ }^{1}$ Die in den Varianten-Verzeichnissen einige Male vorkonmenden Ziffern in [ $]$ bedeuten die im Texte unrichtig gedruckten Zeilenangaben. Die vorhergehende Ziffer giebt immer die richtige Zeilenangabe. 
schnell arbeiten wie er versprochen hatte. - Dieser Aufsatz ist das beste und ausgeführteste was ich Dir von meinen philosophischen Versuchen mitzutheilen habe. Manches andre erwartet mehr Fleiss und ruhigere Musse, um alsdann auch deiner Prüfung unterworfen zu werden. Dass ich über das Princip der Philosophie, über die vollständige Ansicht und den Gebrauch desselben, über die Methode des Fortschritts im Folgern, und über einige naheliegende und wichtige Lehrsätze, mit mir einig geworden sey, werden Dir die einliegenden Blätter zeigen; und ziemlich bestimmt angeben, was $\mathrm{Du}$ von meiner Art zu philosophiren möchtest erwarten können. Nur muss ich Dich um eine etwas anhaltende Aufmerksamkeit und um das günstige Vorurtheil bitten, dass jede einzelne abgebrochne Aeusserung im Ganzen Sinn und Bedeutung haben werde, wenn sie auch für sich allein wenig verspricht. Du wirst viel hinzu denken müssen; denn ich habe mich so kurz als möglich gefasst. - Warum ich an Sch.s Schrift so viel Zeit gewandt? die Veranlassung war Hülsens Schrift, welche ganz in seinem Geiste geschrieben ist, ohne ihn so vollständig und deutlich erscheinen zu lassen; überdiess halte ich Sch.s System, einige Kleinigkeiten abgerechnet, für die möglichst consequente Darstellung des Idealismus. -- Angehängt findest Du Fichte's Noten, die Dich überzeugen mögen, wie wenig Aufmerksamkeit man sich von ihm versprechen dürfe. Ich ward förmlich des Dogmatismus beschuldigt, und nach einer mündlichen Unterredung ebenso förmlich losgesprochen; aber ob meine Abweichungen von F.s eignen Darstellungen bedeutend oder unbedeutend seyen, darüber kein erhebliches Wort! Gerade darüber bedurfte ich der Belehrung am meisten, denn ich halte sie für bedeutend, und Fichte's jetzige sehr veränderte Darstellung der W.l. so gut wie die erste für unmethodisch und undeutlich, und seine darauf sich gründenden Ableitungen im Naturrecht und der Moral, so viel glückliche Gedanken auch einzeln ausgestreut seyn mögen, in den Hauptsachen, wie z. B. in der Theorie von der Anerkennung eines vernünftigen Wesens als eines solchen, und in der Freiheitslehre für falsch." -

Brief Herbarts an Rist: Jena, im September 1796:

„Jetzt bin ich beschäftigt, Schelling und Hülsen, die ich noch immer für Eine Partei halte, sorgfältig zu prüfen. Der letzte, glaube ich, kann nur durch das Studium des ersteren ganz verständlich werden."

Hartenstein urtheilt über die Aufsätze: „Die ganze Arbeit trägt übrigens den Character eines noch nicht abschliessenden, sondern versuchenden Denkens, welches sich aber vollkommen dessen bewusst ist, was es von einer speculativen Untersuchung verlangt, einer charactervollen Bescheidenheit, der es ernsthaft um Belehrung zu thun ist, die sich aber durch unhaltbare Gründe und hohe Worte nicht blenden lässt; und einer Sinnesart, die für jede Behauptung mit unerbittlicher Schärfe nach ihren Gründen fragt". $\mathrm{KL}_{\mathrm{S}} \mathrm{S} \mathrm{CH}$ I, S. xxv.

Manuscript. Das Msc. (42 S. $\left.4^{0}\right)$ zu beiden Aufsätzen befindet 
sich als No. 2053 in der Königsberger Universitätsbibliothek. Dasselbe ist als eine Abschrift des an Fichte gesandten Manuseripts zu betrachten. In dem Msc. sind die vielfachen Abkürzungen Herbarts durch Hartensteins Hand ergänzt worden. Ausserdem rühren von Hartenstein die auf den Abdruck bezüglichen dem Setzer geltenden Bemerkungen am Rande des Manuscriptes her. Ob Herbart beide Aufsätze an F. gesandt hat, lässt sich nicht bestimmt angeben, ist jedoch wahrscheinlich. Hartenstein KL ScH Bd. I, S. xxIv und Bd. III, S. v-vI und SW Bd. XII, S. x nimmt an, dass beide Aufsätze Fichte überreicht worden sind. F. hat nur Bemerkungen zu No. 5 gemacht, welche von $\mathrm{H}$. in Abschrift den beiden Aufsätzen beigefügt worden sind. Die Ueberschrift zu diesen Fichte'schen Bemerkungen: „Fichte's Noten .zu meiner Beurtheilung von Schellings Schriften: über d[ie] M[öglichkeit] e[iner] F[orm] d[er] $\mathrm{Ph}$ [ilosophie] und über das Ich; mit meinen Antworten," lässt annehmen, dass Herbart beide Schriften an Fichte gesandt und dass er bei der Abschrift der Fichte'schen Noten übersehen hatte, dass sich diese Noten nur auf den einen Aufsatz (No. 5) bezogen.

Drucke. Die Aufsätze sammt den Fichte'schen Noten sind abgedruckt KL ScH III, 43-74 u. SW XII, 10-37.

Grundlage. Das Manuscript.

Textveränderungen. Die häufigen Abbreviaturen Herbarts sind im vorliegenden Abdrucke ergänzt. Die Ergänzungen sind in [] gesetzt, sodass der Herbartsche Originaltext leicht $\mathrm{zu}$ erkennen ist. Zuweilen war wegen der Correcturen von Hartensteins Hand die ursprüngliche Abkürzung nicht mehr deutlich zu erkennen. In diesen übrigens irrelevanten Fällen ist die Hartensteinsche Ergänzung gegeben worden. Zur leichteren Orientirung sind in [ ] hinter den Herbartschen Verweisen auf die Paginirung der Originalausgaben von Schellings Schriften die entsprechenden Seitenzahlen der übrigen Ausgaben von Schellings Werken angegeben worden (s. die Anmerkungen auf S. 12 u. 18). Die Fichteschen Noten sind nicht als Anhang gedruckt, sondern aus Rücksicht auf grössere Uebersichtlichkeit immer den bezüglichen Stellen des Textes als Anmerkung unter dem Texte beigefügt worden.

Es ist ferner gesetzt worden:

S. 24. Z. 19 fort .. statt . - fährt (Msc.).

Varianten. S. 15. Z. 30. Mse.: Wir müssten

SW: Wir müssen.

S. 21. Z. 26. Msc.: gar nicht mit gesetzt

SW: gar nicht gesetzt.

S. 24. Z. 12. Msc.: angenommen würde

SW: angenommen wird.

S. 24. Z. 19. Msc.: fährt

SW: fort.

S. 24. Z. 39. Msc.: wann er

SW: wenn er. 
xLvI Vorrede des Herausgebers zum I. Bande.

Varianten. S. 25. Z. 29. Hse.: (bey 15)

SW: bei 15 (Anm. a).

S. 26. Z. 36. Msc.: Rede seyn SW: Rede sein kann.

S. 27. Z. 39. Use.: absolut ist SW: absolit ist.

S. 29. Z. 13. Msc.: an sich geben SW: an sich gebe.

S. 30. Z. 36. Msc.: das willkührlich eingeschoben? SW: das willkührlich Eingeschobene?

S. 31. Z. 2. Msc.: (s. die Veberschrift) $\mathrm{SW}$ : vacat.

S. 31. Z. 8. Msc.: kein $\delta o \chi \varepsilon \iota 0 \nu$ SW: ein $\delta o \chi \varepsilon i o \nu$.

S. 32. Z. 32. Msc.: denn freylich SW: dann freilich.

S. 33. Z. 20. Msc.: schlechthin nicht SW: schlechthin nicht.

S. 35. Z. 5. Msc.: von Idealismus

SW: vom Idealismus.

Paginirung. Unter dem Texte befindet sich die entsprechende Bände- und Seitenangabe von SW u. KL Scr.

6. Ein Augenblick meines Lebens [1796?. S. $37-38$.

Der kleine Aufsatz ist nach einem Spaziergange, den Herbart im Sommer 1796 nach dem Jena benachbarten Dorfe Kunitz unternahm, entstanden. Familienverhältnisse, deren er in einem Briefe an Smidt vom 27. Juni 1796 gedenkt, mögen ibn in die im Eingange erwähnte düstere Stimmung versetzt haben. Nachlasse.

Manuscript. Das Msc. (1 Blatt $4^{0}$ ) befindet sich in Rists

Drucke. Der Aufsatz ist abgedruckt in $\mathrm{KL} \mathrm{SCH}_{\mathrm{C}} \mathrm{I}$, $\mathrm{xxIx}-\mathrm{xxx}$ u. in SW XII, 782-783.

Grundlage. Das Manuscript.

Varianten. S. 37. Z. 12. Msc.: heitre

SW: heitere.

S. 37. Z. 14. Msc.: geahndet

SW: geahuet.

S. 37. Z. 17. Msc.: grüblenden

SW: grübelnden.

S. 37. Z. 20. Msc.: drüber sah ich

SW: drüber hinaus sah ich.

S. 37. Z. 25. Msc.: An der Saale auf dem Wege nach

Cuniz, am Fusse des Genzig's SW : racat. 
Vorrede des Herausgebers zum I. Bande.

Varianten. \&. 38. \%. 7. Issc: selbsterrungene

SW: selbsterworbene.

s. 38. \%. т. Msc.: nicht mehr Einfalt

SW: nicht Einfiltt.

S. 38. Z. 10. Iss.: Indem ich so zu mir redete

SW: Indem ich zu mir und den meinigen redete.

S. 38. Z. 13. Msc.: auf blumigen Wiesen

SW: auf blumigem Rasen.

s. 38. Z. 15. IIsc.: hatt' ich

SW: hatte ich.

S. 38. Z. 20. Iísc.: morschen Nachen

SW: morschen Kahn.

Paginirung. Enter dem Texte befindet sich die entsprechende bïnde- und Seitenangabe von S'W n. KL ScH.

7. Am 4. Juni [1796]. S. 39.

Herbarts Bricf an Smidt rom 27. Juni 1796:

.,Willst Du ein paar Zeilen haben, die ich neulich hinwarf, da ich allein von Weimar zurückging? lch will sie abschreiben und beylegen. Sie machen nicht die geringsten Ansprüche, und sollen Dir weiter nichts bedeuten, als dass ich mich wohl gern zur schönen Kunst erheben möchte, wenn ich nur könnte, und dass ich mich wenigstens des Reichthums meines Freundes werde freuen können."

Hartenstein bemerkt zu 6 u. 7 in $\mathrm{KL} \mathrm{S}_{\mathrm{CH}} \mathrm{I}$, xxix:

„Dass sich jedoch dieser Gührungsprocess nicht etwa blos in der Form einer kalten Kritik (bezieht sich auf No. 4. u. 5) ausarbeitete, sondern dass er aus der Tiefe der Gesinnung kam und das Gemüth des Jünglings, der sich noch durch manches Andere, was nicht hierher gehört, vielfältig niedergedrückt fühlen musste, mit schmerzlicher Innigkeit durchdrang, beweisen ein paar Blätter (bezieht sich auf 6 u. 7) aus dem Jahr 1796, die sich noch erhalten haben."

Manuscript. Das Mse. $\left(1 \mathrm{Bl} .4^{0}\right)$ befindet sich in Smidts Nachlasse.

Drucke. Das Gedicht ist abgedruckt in $\mathrm{KL}_{\mathrm{ScH}} \mathrm{I}$, xxxI. u. SW XII, $783-84$.

Grundlage. Das Manuseript.

Varianten. S. 39. Z. 2. Msc.: Am $4^{\text {ten }}$ Juni

SW: 4. Juni.

S. 39. Z. 38. Msc.: müd'

SW: müde.

Paginirung. Unter dem Texte befindet sich die entsprechende Bände- und Seitenangabe von SW u. KцScH. 
II.

\section{Aus der Hauslehrerzeit in der Schweiz.}

1. Fünf Berichte an Herrn ron Steiger [1797-1798].S. 43-77.

Herbart hatte sich rerpflichtet, alle zwei Monate über seine Erzieherthätigkeit Herrn von Steiger Bericht zu erstatten. Wie viele solcher Berichte entstanden sind, lässt sich nicht feststellen, überliefert sind nur die 5 vorliegenden. $\mathrm{Ob}$ dieselben durchaus in der hier vorliegenden Form übergeben worden sind, war schon Hartenstein zweifelhaft, der dies aus der Beschaffenheit der Manuscripte schloss.

Er ist geneigt für den ersten und zweiten Bericht (nach Hartensteins Anordnung ist der zweite Bericht vorliegender Ausgabe der dritte) das anzunehmen, in Betreff der übrigen Berichte aber zeigt die Beschaffenheit der Manuscripte, welche ,deutliche Spuren von Zusätzen“ erkennen liessen, die Herbart vielleicht nur für sich, um über seine eigenen Gedanken sich klar zu werden, hinzugefügt hatte.

Manuscript. Von den Manuscripten zu den 5 Berichten, welche sämmtlich Hartenstein sowol zur Zeit der Edition von $\mathrm{KLScH}$ als auch der von SW zur Verfügung gestanden hatten, konnte jetzt nur noch die Existenz des Manuscriptes zum ersten Berichte nachgewiesen werden. Es ist in G. Hartensteins Besitz. Leider habe ich dasselbe nicht eingesehen; es ist dies aber kein Nachtheil, da nach Hartensteins brieflicher Versicherung eine von mir wiederholte Vergleichung desselben mit dem Abdrucke in SW nichts weiter hätte ergeben können, als dass die vorkommenden Eigennamen sämmtlich vollständig wiedergegeben seien, während SW nur die Anfangsbuchstaben druckt.

Drucke. Die Berichte sind vollständig gedruckt in SW XI, S. 1-44 unter dem Titel: An Herr von Steiger 1797-1799; in B II, S. 9-46; in W I, S. $11-61$.

In KLScr I sind die Berichte nur auszugsweise edirt worden.

Grundlage. Der Text rorliegender Ausgabe ist Abdruck von SW XI, S. 1-44, jedoch modificirt

1) dadurch, dass im ersten Berichte nach der Angabe von Hartenstein die Eigennamen nach dem Msc. ungekürzt gedruckt worden sind, also Wyttenbach's statt W's und an wiederholten Stellen Ludwig, Carl, Rudolph statt L. C. R.

2) dadurch, dass die Reihenfolge und Datirung der Berichte in vorliegender Ausgabe abweicht von den bezügl. Bestimmungen der Grundlage. Nur ein Bericht, der erste, trägt ein bestimmtes Datum, die ungefähren Data der übrigen müssen aus inneren Gründen erschlossen werden.

Die folgende Tabelle giebt eine Uebersicht über den Unterschied der Reihenfolge und Datirung der Berichte in vorl. Ausgabe und der Grundlage. 
Bericht 1, 4. Nor. 1797 vorl. Ausg. = Bericht 1,4 . Nor. 1797 von SW

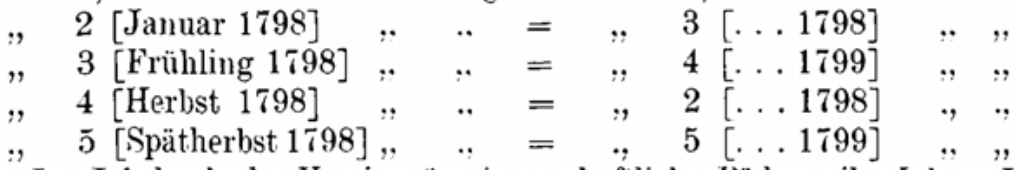

Im Jahrbuch des Vereins f. wissenschaftliche Pädagogik, Jahrg. II 1870 , S. $247 \mathrm{ff}$. ist zuerst ron Dix auf eine ron SW abweichende Reihenfolge und Datirung der Berichte hingewiesen worden und es sind daselbst ausführlich die Gründe für diese Abweichung dargelegt worden. Die ron Dix vorgeschlagenen Aenderungen hat Bartholomäi in seiner Ausgabe (B) der pädagogischen Schriften Herbarts durchweg acceptirt, während Willmann nur in Bezug auf die Reihenfolge Dix folgt, in der Datirung aber ron ihm abweicht.

Der erste Bericht ist nicht in Bern, sondern auf dem Lande, auf dem eine Stunde von Bern entfernt liegenden Steigerschen Landsitz Märchligen, wo sich die Steigersche Familie im Sommer aufhielt, verfasst worden. Wenn Dix a. a. 0. S. 247 die Vermuthung aufstellt, dass Herbart schon im Juli oder August unterrichtet haben muss, so lässt sich jetzt nit Gewissheit feststellen, dass Herbart dies bereits im Juni gethan hat; denn in einem Briefe an Rist rom 15. Juni 1797 giebt er bereits (in Crtheil über die Fähigkeiten seiner Zöglinge. Auch aus Smidts Erinnerungen (ef. S. XIII) geht hervor, dass Herbart im Sommer in voller Thätigkeit war. Es wird sich demnach der erste Bericht auf die Monate Juni bis Ende October erstrecken. Wenn man freilich annimmt, dass gleich mit dem Beginne von Herbarts Thätigkeit die Bestimmung getroffen wurde, dass derselbe alle 2 Monate einen „Bericht" abliefern sollte, so muss bereits dem vorliegenden jetzigen ersten Berichte einer roraufgegangen sein.

Der zweite Bericht fällt, da Herbart auf pünktliches Einhalten der Telmine achten wollte, in den Januar 1798. Welches ist aber nun dieser im Januar 1798 ahgefasste zweite Bericht? Jedenfalls nur der in SW als dritter aufgeführte. In diesem Berichte spricht Herbart sein tiefstes Bedauern darüber aus, dass der „Plan“, welchen er für die Erziehung seiner Zöglinge entworfen hatte und den er für so sicher als möglich hielt, der ihm, wenn derselbe zwei Jahre aufs Strengste beobachtef́ worden wäre, ,eine dauerhafte Wirkung“ versprach, so plötzlich umgestossen worden sei. Der noch nicht 14 jährige Zögling Ludwig trat nämlich als Freiwilliger in das eidgenc̈ssische Heer. „Was soll doch ein junger Mann, der ins Feld geht, mit Chemie und Botanik" (S. 58-59). In dem Bericht ist ferner hervorgehoben, dass auch die Erziehung der anderen Zïglinge mannigfache Störung erfahren, unter anderem auch durch das Helumlaufen, um Bonaparte zu sehen. Bonaparte war im November 1797 durch Bern gereist. Es geht hieraus zur Genüge hervor, dass dieser Bericht, der in SW als dritter aufgeführt wird, als zweiter gelten muss. Da im Februar 1798 Ludwig HEBBAKTs Werke I. 
aber bereits im Felde ist, wie aus einem Briefe Herbarts an Smidt von Ende Februar 1798 hervorgeht, so muss die Abfassung dieses zweiten Berichtes auch aus diesem Grunde in den Januar gesetzt werden. Herbart hat also den Termin der Ablieferung pünktlich eingehalten.

Der dritte Bericht [Frühling 1798] ist in SW als vierter bezeichnet und in das Jahr 1799 verlegt worden. Dass aber dieser dritte Bericht nicht lange nach dem zweiten gesetzt werden kann, geht aus folgenden Umständen hervor. Ludwig, welcher mit dem eidgenössischen Heer ins Feld gezogen war, ist zurückgekehrt. Das Bernerische Heer war Anfang März geschlagen, Bern selbst bald darauf von den Franzosen eingenommen worden. „Er (sc. Ludwig) war schon etwas gewesen (d. h. Soldat), jetzt wollte er wenigstens wissen, was er kūnftig sein werde." - Der Bericht spricht hauptsächlich von Rudi und Karl, dies ist nur dann nicht auffallig, wenn er Rechenschaft giebt über eine Zeit, in der Ludwig grösstentheils abwesend war. Das war aber im Februar bis Anfang März der Fall. In dem Bericht schreibt Herbart: „Meine Stimmung war den ganzen Winter zu düster, ein Gedanke raubte mir oft das Bewusstsein aller meiner anderen Verhältnisse." Herbart war im Winter 1797-98 mit den Problemen der mathematischen Psychologie beschäftigt und hierauf wird sich die obige Bemerkung beziehen. „Erst im Oberlande fühlte ich, was ich bei meinen Zöglingen vermöchte (S. 64). Während der Kriegszeit hatte die Steigersche Familie Bern verlassen und war ins Oberland gezogen. An Halem schreibt Herbart am 28. Januar 1798: „Meine Sachen habe ich einpacken müssen, damit wir - die Frau und ich mit den jüngeren Kindern, bey dem täglich befürchteten Einfalle der Franzosen gleich nach dem Oberlande hin aufbrechen können".

Der Bericht fällt demnach in das Frühjahr 1798.

Der vierte Bericht [Herbst 1798] ist in SW der zweite. Für die Anordnung und Datirung in vorliegender Ausgabe sprechen folgende Umstände. Es wird in dem Berichte davon gesprochen, dass ,seit einem Jahre" mit Ludwig Mathematik, mit dem Kleinen die Odyssee getrieben worden ist. Im Frühjahr hatte Herbart den moralischen Unterricht mit Ludwig angefangen (S. 69), freilich hatte er ihn nicht in der Weise ertheilen können, wie er seinem ursprünglichen Plane gemäss, der durch die Betheiligung Ludwigs am Berner Feldzuge nicht zur Ausführung gelangt war, ihn gern ertheilt hätte. Nachdem er sich von der Erfolglosigkeit seines Unterrichtes überzengt hatte, musste er es für das Gerathenste halten, denselben ganz zu sistiren. Dies kann im Sommer 1798 gewesen sein. Nach dem vorliegenden Berichte nun hat er den Unterricht, nachdem in Ludwigs Denkungsweise Aenderungen eingetreten sind, wieder aufgenommen. Es wird weiter bemerkt, dass L. während des bevorstehenden Winters 3 Stunden Religionsunterricht bei $\mathrm{H}$. N. als Vorbereitung zu seiner Confirmation empfangen soll. Dieser bevorstehende Winter ist der Winter 1798/1799. 
Der Bericht wird im Herbst, vielleicht im September 1798 abgefasst sein.

Der fünfte Bericht vorliegender Ausgabe ist auch der fünfte Bericht in SW, wird aber hier in das Jahr 1799 verlegt. Diese Datirung kann nicht die richtige sein. Dix a. a. O. S. 251 ist zweifelhaft, ob der Bericht in den Herbst oder Winteranfang des Jahres 1799 gehört. Bartholomäi verlegt ihn ohne Angabe des näheren Datums auch in das Jahr 1799; ebenso Hartenstein in SW. Willmann dagegen ist geneigt ihn in den Spätherbst $1798 \mathrm{zu}$ verlegen. Dieser Annahme ist die vorliegende Ausgabe gefolgt. Für diese Datirung sprechen folgende Umstände. „Kaum ein Jahr war Karl der Gegenstand meiner vorzüglichen Sorge." Das wurde er gegen Anfang des Jahres 1798, als Ludwig in die eidgenössische Armee eintrat. Herbart kommt in dem fünften Berichte auf seine Verabredung zurück, die er in Betreff seiner freien Zeit mit Herrn von Steiger vor $1 \frac{1}{2}$ Jahren getroffen hatte. Wenn diese Verabredung, wie es sehr wahrscheinlich ist, in die Zeit des Beginnens der Erzieherthätigkeit fällt, so würde der Bericht in den Spätherbst, vielleicht Winter des Jahres 1798, zu setzen sein.

Dass der Bericht aber nicht in den Herbst oder Anfang des Winters 1799 zu setzen sei, dürfte auch daraus hervorgehen, dass nach dem Berichte Herbart mit frischem Muthe an die Arbeit, von Neuem ans Werk geht und dabei von einem zunächst zu befolgenden Plane spricht; während er nach den Briefen an Smidt und Böhlendorf vom 4. und 28. September 1799 bereits an die Lösung seines Verhältnisses zur Steiger'schen Familie denkt. Er bittet in dem bezüglichen Briefe seinen Freund Smidt, derselbe möge sich nach einem geeigneten Nachfolger für ihn umsehen. Er habe zwar Herrn von Steiger selbst seinen Entschluss, die Erzieherthätigkeit aufzugeben, noch nicht mitgetheilt, indess habe er doch bereits seine beiden Freunde Muhrbeck und Böhlendorf beauftragt, sich nach einem Nachfolger für ihn umzusehen. In dem Briefe an Böhlendorf vom 20. September fragt er diesen, ob er einen Nachfolger für ihn gefunden habe.

In dem vierten Berichte, der in den Herbst, vielleicht September $1798 \mathrm{zu}$ verlegen war, spricht Herbart davon, dass er ,ohne Scheu“ daran denke, in Zukunft Herodot, Xenophon, Plato, einige Stücke der Tragiker, Tacitus etc. mit Karl zu lesen. In dem fünften Berichte ist aber nur Homer erwähnt. Aus dem Briefe an Smidt vom 4. September 1799 erfahren wir dagegen, dass er mit Ǩarl nicht nur Homer, sondern auch Sophokles und Plato liest. Wäre der fünfte Bericht in das Jahr 1799 zu verlegen, so würde also sicher auch von Plato und Sophokles die Rede sein. Uebrigens sollte nach dem vierten Berichte ja auch erst im „Frühjahr" die Odyssee von einem anderen griechischen Schriftsteller abgelöst werden. So bleibt nur das Jahr 1798 übrig und zwar, wenn der vierte Bericht im Herbst (September) fällt, der November als Zeit der Abfassung übrig. Wenn aber im September der vierte, Ende 
November der fünfte Bericht abgefasst worden ist, so ist auch nicht auffällig, dass der letztere etwas dürftig geworden ist. Es ist eben innerhalb des kurzen Zeitraumes, der zwischen der Abfassung des vierten und fünften Berichtes verflossen ist, eine wesentliche Aenderung nicht eingetreten.

Textveränderangen. Ausser den in der Rubrik "Grundlage“ erwähnten Textesveränderungen sind noch die folgenden zu rerzeichnen. Es ist gesetzt worden:

S. 70. Z. 17. Anlage gewährt... statt Anlage gewährte SW B.

S. 72. Z. 13. soll, rorzubereiten ... statt sollen, vorzubereiten SW B. Diese Emendationen hat auch Willmann, der sie ohne besondere Anmerkungen lediglich durch [] kennzeichnet (cf. S. 70 u. S. 72).

Paginirung. In dem Texte ist die entsprechende Paginirung von SW, des Grundtextes, unter dem Texte, die der Ausgaben B und $\mathrm{W}$ augegeben worden.

\section{Gebete für die Steigerschen Knaben. S. 78-82.}

In dem ersten Berichte an Herrn von Steiger spricht Herbart von den Gebeten, die er für seine 3 Zöglinge „schon ehemals", also gleich im Anfange seiner Erzieherthätigkeit abgefasst und Herrn von Steiger übergeben habe. Jetzt (also im November 1797) will er dieselben seinen Zöglingen überreichen. Bei Ludwig sollen sie zur Unterstützung des Unterrichtes in der Moral dienen. (S. S. 50).

Manuscript. Die Manuscripte (lose Blätter) zu den Gebeten $1 \mathrm{u.} 2$ (S. 78 u. 79) sind in dem Besitze des Herrn A. v. Steiger in Bern, die zu den Gebeten 3-8 in dem Besitze des Herrn Prof. Dr. Hartenstein in Jena.

Drucke. Die Gebete 1 u. 2 sind zuerst gedruckt in HR S. 52-53 und von da übergegangen in die Ausgabe W I, S. 19-20 (Anmerkung). Die übrigen Gebete (3-8) waren noch ungedruckt.

Grundlage. Dem Drucke haben die Manuscripte als Grundlage gedient.

Textveränderungen. S. 79, Z. 37. Ich danke Euch auch ... statt ... Ich danke ich Euch.

Paginirung. $\mathrm{Zu}$ den Gebeten 1 u. 2 ist die entsprechende Paginirung von $\mathrm{HR} u$. W unter dem Texte angegeben worden.

3. Bericht über eine Reise in die Alpen [1798]. S. 83-92.

Manuscript. Das Manuscript zu diesem Berichte, im Besitze der Königsberger Universitätsbibliothek (Ms 2075) besteht aus $16 \mathrm{~S} .4^{0}$ (S. 16 leer).

Druck. Der Bericht ist bisher nur abgedruckt in HR S. 73-85.

Grundlage. Das Manuscript. 


\section{Textveränderungen.}

S. 84. Z. 40. Lütschine (HR) . . statt Lütschinen.

S. 91. Z. 19. Sinne (HR) ... statt Simnen.

S. 92. Z. 14. ins Thal öfnet $(\mathrm{HR})$... statt. . . ins Thal öffnen.

S. 92. Z. 18. ferner, den Leser (HR) . . . statt . . . ferner, denselben.

Varianten. S. 83. Z. 13. Isse: Wengernalp HR: Wangernalp (ebenso S. 84, 86, 88, 90).

S. 84. Z. 18. Msc.: ging HR: gingen

S. 84. Z. 37. Msc.: letztre HR: letztere

S. 84. Z. 40. Issc: Lütschinen HR: Lütschine

S. 87. Z. 1. Msc.: einiges hölzernes Geräthe HR: einiges hölzerne Geräth

S. 89. Z. 7. Isc.: Er verfolgt HR: Er verfolgte

S. 89. Z. 32. Msc.: gesehn

HR: gesehen.

S. 89. Z. 36. Isc.: IIettenberg HR: Wettenberg.

s. 90. Z. 1. Isc.: Karh

HR : Carl

S. 90. Z. 40. Msc.: wornach

HR: wonach

S. 91. Z.4--5. Msc.: Unterwegens

HR: Unteriegs

S. 91. Z. 9. Mse.: Herrn

HR: Herren

S. 91. Z. 19. Mse: Simnen

HR: Sinne

S. 92. Z. 14. Mse.: Thal öffnen.

HR: Thal öffnet.

S. 92. Z. 18. Msc.: ferner, denselben an

HR: ferner, den Leser an

S. 92. Z. 35. Isc.: Thun wieder nach

HR: 'Thun nach.

Paginirung. Unter dem Texte ist die Paginirung von HR angegebien.

4. Ueber philosophisches Wissen und philosophisches Studium [1798]. S. $93-105$.

5. Erster problematischer Entwurf der Wissenslehre. Engisstein, Ende August 1798. S. 106-122. 
In einer Replik, welche Herbart in der Jenaer Literatur-Zeitung Jahrg. 1809, Intelligenz-Bl. 26. S. 222 gegen eine Recension seiner Allgemeinen praktischen Philosophie (Halle'sche Literatur-Zeitg., 1809, No. 40) veröffentlicht, rerwahrt er sich gegen den ihm gemachten Vorwurf der „Raschheit"“, indem er darauf hinweist, dass er bereits in den Jahren 1798 und 1799 die Grundgedanken seiner Metaphysik festgestellt habe.

Sowol aus den „Berichten" an Herrn ron Stejger als auch aus dem Briefwechsel erfährt man, dass das speculative Interesse Herbarts trotz der anstrengenden Erzieherthätigkeit mit immer grösserer Intensivität herrortrat.

Herbarts Brief an Rist rom 12. Juni 1797: „In Jena war ich in der letzten Zeit zu träge oder zu dumm, meine Wissenschaftslehre förmlich und ordentlich fortzuführen, zu stolz, um andere Beschäftigungen in ihre Stelle zu setzen, zu arm an Mannigfaltigkeit der äusseren Verhältnisse, um im Leben das Bedürfniss eines sicheren, ganz geprüften, aller Wege kundigen Führers - so etwas soll doch wohl ein phil. System sein - tief genug zu fühlen."

1798 am 28. Januar schreibt er an Halem: Weder vor der grossen Natur, noch vor der Arbeit, die ich hier gefunden habe, kann in mir das Bedürfniss derjenigen Philosophie verstummen, die ich suchte und zu der ich den Eingang gefunden zu haben glaube. So oft ich staunend zurückkehre ron dem Anblick, wie hier die Natur die äussersten Enden des Schönen und Erhabenen in Ein Unnennlsares verwebt hat, so oft die Pflicht von mir heischt, ich solle mit Lehre und Empfindung in die Tiefe menschlicher Herzen eindringen: fühle ich mich gewaltiger hingerissen gegen die unbekannte Einheit ausser mir, die alles das zusammenhält und belebt; und die unbekannte Kraft in mir und andern, die es im Bilde zusammenfasst, und dem Bilde selbst Sinn und Bedeutung giebt: Es dünkt mich ein gutes Zeichen für meine Idee der Wissenschaftslehre, dass sie sich allenthalben wieder aufdrängt. Von Fichte's bisherigen Ausführungen gestehe ich, dass sie mir oft nur durch den Contrast das Ideal zu erheben scheinen."

1798, im Februar, schreibt er an Simidt: „Willst Du mich sehen, so siehst $\mathrm{Du}$ mich in meiner Werkstätte. Bestäubt, schwitzend; vielleicht keuchend, ermüdet - doch wieder ansetzend, und Etwas fördernd. Zuweilen lege ich die Arbeit aus der Hand, sehe gen Himmel und es ist mir unbeschreiblich wohl." -

In dem dritten „Bericht“, dessen Abfassung in vorliegender Ausgabe in das Frühjahr 1798, also kurze Zeit nach dem obigen Brief gesetzt worden ist, schreibt er: „Leberhaupt raubt mir oft ein Gedanke das Berwusstsein aller meiner andern Verhältnisse, leider mehr durch das Streben ihn zu ergründen, als durch seine Lebhaftigkeit."

Eine deutliche Bezugnahme auf die philosophischen Forschungen, welche Herbart in den vorliegenden Aufsätzen niedergelegt hat, findet 
sich in dem ausführlichen Briefe an seine Eltern, Bern, den letzten Juni 1798: „Mein jetziger Reichthum besteht in einigen Ueberzeugungen, die den Keim vieler folgenden zu enthalten scheinen. Sie sind gewonnen in drittehalb Jahren einer Musse, wie ich sie in meinem Leben nicht wieder erwarten darf, wo die Empfänglichkeit und Lebhaftigkeit des jugendlichen Geistes sich mit Umständen, mit einer Umgebung von Lehrern und Freunden rereinigte, die mir Muth und Zutrauen zu dem gaben, was damals in mir erwachte. Aber Gedanken erzeugen entweder immer neue oder veralten und rerschwinden. Jetzt erhebt mich eine innere Gewissheit über die Systeme unserer Zeit, das Fichte'sche so wenig, als das Kant'sche ausgenommen; sollte ich auch irren, so halte ich es doch für ein grosses Glück, ohne Führer und ohne Furcht ein eigenes Feld durchwandern zu könneu, das sich bei jedem Schritte zu erweitern scheint“... . „Fichte's wiederholte Zengnisse und wohl mehr noch die Proben, die ich mir selbst abgelegt habe, scheinen mich zu rersichern, dass, wenn mir irgend etwas gelingen könne, es die Speculation sei.

Befriedigen mit dem, was unsere berühmten Männer geleistet haben, kann ich mich unmöglich; selbst die Richtungen, die sie nehmen, entfernen sich weit ron dem Wege, der ziemlich bestimmt rorgezeichnet, als derjenige ror mir daliegt, auf dem man sich zunächst versuchen sollte“ ..... „Meine Philosophie - - lassen Sie mich das Wor übersetzen, damit es nicht hart klinge -... mein Streben nach Wahrheit -.. will sich nicht blos unter Idealen herumtreiben, es möchte vor allen Dingen begreifen -- also auch sehn, aber nicht blos sehn --- was der Mensch ist, wie er es ward, und wie er es mehr werden kann: - es ist dabei viel zu schüchtern im dunkeln Reiche der Abstraction, als dass es nicht gern allenthalben bei der Erfahrung und Geschichte Bewährung und Bestätigung suchen sollte."...

Directer noch als die vorhergehenden Briefe bezieht sich dẹ Brief Böhlendorfs an Rist rom 10. Dec. [September?] 1798 auf die rorliegenden Aufsätze:

„Herbart hat sein System gefunden. Lache nur nicht; es ist sehr ernstlich gemeint. Ich bin zwar selbst noch keinem philosophischen System zugethan, aber dennoch könnte es leicht sein, dass ich und Steck, die wir beide eine Stunde wöchentlich Herbart philosophiren hören, von dem neuen Propheten besiegt würden. Dass es kein System, wie von Reinhold, Kant, Fichte, Schelling, sondern eine ganz andere Art von Systemen sei, kann Dich schon seine Entstehung lehren. Fichte hat die Wissenschaftslehre zuerst im Traume gesehen; Herbart hingegen, - nachdem er sich durch Fichte's und Schelling's, Kant's Systeme durchgearbeitet, Chemie, Mathematik als schwere Steine langsam vor sich hergewälzt, und mit einer gewissen selbstbewussten Macht in der Welt um sich her gesehen, dann in sein eignes Herz zurückgesehen, entstand das seinige in dem anmuthigen Wäldchen von Engistein, 
unweit Höchstetten, wo er drei Wochen noemitisirte; und ein solches System, in der freien Natur entstanden, verschmäht die Anhänglichkeit freier Naturen nicht. Wir selbst sind selbstredend jetzt nur noch im Vorhofe begriffen; wenn wir ins innere Heiligthum gelangen, so soll Dir das Deinige nicht vorenthalten werden. Für jetzt will ich Dich nur zur Taufe eines Kindes eingeladen haben, das den Genius des Gedankens zum Erzeuger, die Natur zur Mutter, die Freundschaft zur Säugamme gehabt hat."

Herbart schränkt in bescheidener Weise das begeisterte Urtheil Böhlendorfs in dem Briefe, der zugleich mit dem Böhlendorf'schen an Rist geht, ein :

„Was ich gearbeitet, hat Dir Böhlendorf richtig angegeben, wenn Du statt eines Systems einige erste Punkte davon denkst, deren Unrichtigkeit ich beim weitern Auszeichnen noch nicht gefunden habe. Mir wäre das an sich noch nicht der Rede werth gewesen, und Du wirst es hoffentlich keiner weiteren Rede werth halten. Kaum kann es bis jetzt die Freunde interessiren, deren mündliches Urtheil mich berichtigen kann."

Es ist anzunehmen, dass Böhlendorf durch die Worte: Wir sind selbstredend jetzt nur noch im Vorhofe begriffen, an den Wortlaut des Aufsatzes No. 4, S. 93 gedacht hat. -- Die Anmerkungen (S. 114-122) zu No. 5 hat Herbart auf jeden Fall kurze Zeit nach der Abfassung des Aufsatzes niedergeschrieben.

Drucke. No. 4 in HR.; No. 5 in SW XII, S. $38-57$ rollständig, theilweise in KL ScH I, xuII ff.

Grundlage. Für No. 4 der Text von HR, für No. 5 der Text von SW XII.

Textveränderungen: $N_{0} .4$ vacat. No. 5 :

S. 112, Z. 41 andre non B . . . statt . . andre von B.

S. 113, Z. 11 Gedanke (Alle blosse ...) statt . . . Gedanke (Blosse . . .).

S. 118, Z. 1 vom Reflectirten . . statt.. vom Reflectiren.

Ausserdem wurde bei den Verweisungen, die innerhalb der dem Aufsatze No. 5 hinzugefügten Anmerkungen auf einzelne Sätze des Aufsatzes gemacht wurden, die entsprechende Paginirung vorl. Ausg. substituirt.

Paginirung. Im Texte von No. 4 ist die entsprechende Paginirung von HR, im Texte ron Nr. 5 diejenige von SW angegeben worden.

\section{III. \\ Aus dem Jahre 1800.}

1. Zur Kritik der Ichvorstellung. S. 125-127.

2. Ueber den Unterschied ron Kantischem und Fichteschem I.dealismus. S. 128. 
3. Ueber das Bedürfniss der Sittenlehre und Religion in ihrem Verhältniss zur Philosophie. S. 129-141.

In seinem 4. Berichte an Herrn ron Steiger vom Herbst 1798 bittet Herbart für das nächste Jahr (1799) um die Begünstigung sich nach den 6 Unterrichtsstunden in sich selbst zurückziehen zu dürfen, nachdem er vorher erklärt hat, dass er Arbeiten vor sich sähe, deren grösste Schwierigkeiten zwar schon überwunden schienen, die er aber „ganz durchdringen“ müsste, wenn er ,zur völligen Ruhe und Besinnung kommen" sollte. Die vorliegenden Aufsätze, deren Abfassung unmittelbar nach der Hauslehrerzeit in die Zeit des Bremer Aufenthalts fällt, können als Bestätigungen der angedeuteten philosophischen Forschungen angesehen werden; sie beweisen, dass die in der Wissenslehre erörterten Probleme ihn unausgesetzt beschäftigten, dass die Gedanken in ihm nach Klarheit rangen.

Dracke. Die 3 Aufsätze sind abgedruckt in HR S. 247--275.

Grundlage. Der Text von HR.

Textveränderungen. S. 126, Z. 25. des Denkens . . statt . . der Denkens.

Paginirung. Die in [ ] gesetzten Ziffern im Texte bezeichnen die entsprechende Paginirung von HR.

\section{IV.}

\section{Ideen zu einem pädagogischen Lehrplan für höhere Studien [1801]. S. $143-152$.}

Smidt, welcher im Jahre 1800 in dem jugendlichen Alter von 27 Jahren Senator in Bremen geworden war, richtete in dieser Stellung sein Hauptaugenmerk auf die geistige und materielle Hebung seiner Vaterstadt. Vor Allem war es zunächst das Schulwesen, das er zu organisiren sich vornahm. Es war selbstrerständlich, dass Herbart sich an den über die Reform stattfindenden Discussionen betheiligte. Der vorliegende $\Lambda$ ufsatz ist in Rücksicht anf diese Reformen jedenfalls nach einer bezüglichen Aufforderung Smidts geschrieben. Aus dem Aufsatze selbst geht hervor, dass Herbart zur Durchführung seiner "Ideen“ an die Uebernahme eines Lehramts bei der Stadt Bremen dachte. Er will seinen „Plan“, der sich „sehr wohl mit einer allmähligen stufenweisen Einführung" vertrüge unter der Voraussetzung, dass ihm 12 Stunden zur freien Disposition zugesichert würden, zur Ausführung bringen. Mit Professor Rump habe er bereits darüber conferirt. Eine Lehrerstelle an einer öffentlichen Anstalt Bremens hat Herbart, wie genaue Nachforschungen ergeben haben, nicht bekleidet.

Manuscript. Das Msc. $\left(6 \mathrm{Bl} .4^{\circ}\right)$ ist im Besitze der Oldenburger Realschulbibliothek. (Philos. 8. 1.) 
Drucke. Der Aufsatz ist abgedruckt in HR S. 276-284, R II, 337-343 und $\mathrm{W}$ I, 74-82.

Grundlage. Der Text des JIanuscriptes ist abgedruckt worden.

Textveränderungen. S. 150. Z. 33 mit betrïchtlich vermehrtem . . . statt mit beträchtlich vermehrten.

Varianten. S. 145. Z. 7 [ 8 ]. Msc.: Aus elen diesen

HR: In eben diesen $\mathrm{R}, \mathrm{W}$.

S. 145. Z. 28. Msc.: Fs musste

HR: Es müsste R. W.

S. 146. Z. 15. Msc.: entstehn

HR: entstehen.

So auch Msc. S. 146. Z. 16 ,,übergehn“, S. 147, 2 ,seltner“, Z. 23 ,andre", 149, 1 „seltne“, 149, 20 u. 150, 26 „angegebne“", 151 ,insbesondre", in welchen Fällen HR sowie R, W immer das $e$ einschieben, also: übergehen, seltener, andere etc.

S. 148. Z. 25. Msc.: Leuten nämlich, die

Hh: Leuten, die R, W.

S. 148. Z. 27. Msc.: benutzen

HR: benützen $\mathrm{R}, \mathrm{W}$.

S. 148. Z. 34-35. Msc.: werden mussten

HR: werden müssen $\mathrm{R}, \mathrm{W}$.

S. 148. Z. 35. Msc.: hierauf folgen lässt

HR: hier auf jene folgen lässt W.

( $R$ druckt: hier auf auf jene).

S. 149. Z. 13. Msc:: Interesse abgewönnen

HR: Interesse abgewännen R, W.

S. 149. Z. 21. Msr.: Weise benutzte. R. W.

HR: Weise benützte.

S. 149. Z. 28. Mse.: vestzuhalten („rest" gesperrt)

HR: festzuhalten (,fest" nicht gesperrt) $R, W$.

S. 150. Z. 32. Msc.: neuen Sprachen

HR: neuern sprachen $\mathrm{R}, \mathrm{W}$.

S. 150. Z. 40. Msc.: Vergessenheit geriethe

HR: Vergessenheit gerathe R, W.

S. 151. Z. 26. Msc.: noch meine (,meine" gesperrt)

HR: noch meine (,meine" nicht gesperrt) $\mathrm{R}, \mathrm{W}$.

S. 151. Z. 32. Msc.: Kabinetter

HR: Cabinete R. W.

S. 152. Z. 12. Msc.: hievon

HR: hierron R. W.

S. 152. Z. 13. Msc.: Lat.

$\mathrm{HR}$ : lateinischen $\mathrm{R}, \mathrm{W}$. 
Ferner weicht $W$ an folgenden Stellen von Msc. und $H R$ ab, indem diese Ausgabe S. 146, Z. 13 ,Gründliches“ nur gesperrt, statt gesperrt und fett druckt, und sie S. 147, Z. 42 ,unter anderm" setzt statt ,.unter andern".

Paginirung. Unter dem Texte ist die entsprechende Paginirung von $H R, R$ u. W angegeben.

\section{V. \\ Ueber Pestalozzis neueste Schrift: Wie Gertrud ihre Kinder lehrte. An drei Frauen. [1802.] S. 153-163.}

Herbart an G. A. r. Halem, Bremen, Anfang May 1801:

„Sie möchten wol einen Versuch ron mir darauf ansehn, ob er in die Irene passt? Das Thema: Geist der pestalozzischen Erziehung, reizt mich sehr, und mit Hülfe der Nachrichten meines Freundes Ziemssen gelänge es mir vielleicht - weun es anders, nach dem ron $\mathrm{P}$. selbst jetzt herauskommenden Werke, einem andern noch erlaubt seyn kann, seinen Geist darstellen zu wollen".

Der vorliegende Aufsatz ist der in Aussicht gestellte Versuch. Er erschien 1802 in der ron G. A. von Halem redigirten Mionatsschrifi „Irene“" (J).

Der Aufsatz trägt in der Irene den Haupttitel: „Ueber Pestalozzi“ und zerfällt in 2 Abtheilungen, deren 1. die Ueberschrift: ,A Auszug eines Schreibens an den Herausgeber" und deren 2. den Titel: „Ueber Pestalozzis neueste Schrift: Wie Gertrud ihre Kinder lehrte" trägt. Dieser 2. Titel ist in den spätern Abdrücken in $\mathrm{SW}, \mathrm{KL} \mathrm{ScH}, \mathrm{B}, \mathrm{R}, \mathrm{W}$ der Haupttitel geworden und ist auch, da der Aufsatz überall unter diesem Titel eitirt wird, in die vorliegende Ausgabe herübergenommen worden.

Die 3 Frauen, denen er gewidmet ist, sind die Frau Smidts, Frau Noltenius, Frau Senator Castendyk (s. S. xv).

Der Brief Herbarts (rom 24. Dec. 1801), welcher das Manuscript des Aufsatzes begleitete, ist von Halem, wie schon aus der Ueberschrift: „Auszug etc." hervorgeht, nur bruchstückweise und auch dieses Bruchstück nicht diplomatisch genau abgedruckt.

Der erste in der Irene fehlende Abschnitt des Briefes, welcher übrigens Bezug auf den Aufsatz hat, lautet: „In Eile sende ich Ihnen, mein hochgeschätzter Gönner und Freund, einen Aufsatz, der in aller Langsamkeit endlich so weit gekommen ist, Ihnen für die Irene, oder doch zu Ihrer nachsichtsvollen Durchsicht, rorgelegt werden zu können. Die Schuld dieser Langsamkeit liegt nicht an mir. Mein Freund Ziemssen in Bern hat mich von Ostern an auf nähere Nachricht von Pestalozzi hoffen, und warten lassen, und ist endlich darüber krank geworden; Pestalozzi's 
Schrift, wie Gertr. ihre K.l., erwartete ich ebenfalls weit früher; als sie erschien, habe ich sie sogleich durchgearbeitet, unmittelbar darauf den einliegenden Aufsatz geschrieben, und ihn die Kritik der Frauen, denen er gewidmet ist, passiren lassen. Darauf aber bin ich wochenlang von denselben Frauen, die sich Abschriften davon nehmen lassen wollten, - so wie diese von ihren Copisten hingehalten worden; endlich vor einer Stunde kommt mein Exemplar wieder zu meinen Händen; und nun schreibe ich Ihnen diesen Brief in Gegenwart des Herrn Walte, der neben mir rechnet. Es wird mich freuen, wenn Sie meinem Versuche die Aufnahme nicht versagen wollen. Die Pestalozzische Unternehmung scheint mir für Deutsche gar sehr einer eigentlichen etc. etc."

Manuscript. Nur von dem Briefe ist noch das Msc. in dem auf der Oldenburger Bibliothek befindlichen Briefwechsel Halems vorhanden.

Drucke. Der Aufsatz ist abgedruckt in der Irene $(J)$ Bd. I (1802) $\mathrm{S} .15-51$, von hier ist er übergegangen in die Ausgaben SW XI, S. $45-60$, Ku Scr III, 74-90, B II, 53-66, R II, 245-258, W I, 83-100. In der Ausgabe B fehlt der Brief.

Grandlage. Der Text der Irene (J).

Textveränderungen. S. 165 . Z. 21 werden könnte ... statt ... werden konnte.

\section{Varianten.}

S. 155. Z. 10. J: scheint mir für Deutsche

SW: scheint für Deutsche.

S. 155. Z. 17-18 J: Leserinnen der Pestalozzisehen Schrift, die den Müttern etwas unbehutsam gewidmet zu seyn scheinet (SW, KL Scr, R, W)

Msc.: Leserinnen der, den Müttern etwas unbehutsam gewidmeten Pestalozzischen Schrift.

S. 155. Z. 18. J: scheinet

$\mathrm{SW}$ : scheint $\mathrm{KL} \mathrm{ScH}, \mathrm{R}, \mathrm{W}$.

S. 156. Z. 28. J: bestimmt haftend

SW: bestimmt heftend $\mathrm{KL} \mathrm{ScH}, \mathrm{B}, \mathrm{R}, \mathrm{W}$.

S. 156. Z. 31 . J: eines

SW: eins $\mathrm{KL}_{\mathrm{L}} \mathrm{CH}, \mathrm{B}, \mathrm{R}, \mathrm{W}$.

S. 156. Z. $32-33$ J: meinem Ohre

SIV: meinen Ohren $\mathrm{KLSSH}_{\mathrm{L}} \mathrm{B}, \mathrm{R}, \mathrm{W}$.

S. 157. Z. 40 . J: Erfahrungen

SW: Erfahrung KL Sch, B, R. W.

S. 158. Z. 28. J: ob es auch

SW: ob das auch KLScH, B, R, W.

S. 158. Z. 34. J: In weiterm Gespräche

SW: Im weiteren Gespräche $\mathrm{KL} \mathrm{ScH}, \mathrm{B}, \mathrm{R}, \mathrm{W}$.

S. 159. Z. 5. J: in einander fugen und kütten

SW: in einander fügen und ketten $\mathrm{KL} \mathrm{ScH,} \mathrm{B,} \mathrm{R,} \mathrm{W.}$ 
S. 159. Z. 8. J: das blosse Zeichen

SW: ein blosses Zeichen $\mathrm{KL} \mathrm{ScH}_{\mathrm{L}} \mathrm{B}, \mathrm{R}, \mathrm{W}$.

S. 159. Z. 32-33: ist in $J$ ein Absatz, der aber in SW, sowie auch in KL ScH, B, R, W fehlt.

S. 159. Z. 39. J: Lehrart zu steif

SW: Lehrart zu roh B, W

Lehrart zu früh $\mathrm{KL}_{\mathrm{L}} \mathrm{SH}, \mathrm{R}$.

S. 160. Z. 2. J: umfasse ihm

SW: umfasse ihnen SW, KuScH, B, R, W.

S. 160. Z. 11 . J: die Einrichtungen

SW: die Einrichtung $\mathrm{Ku}_{\mathrm{SCH}}, \mathrm{B}, \mathrm{R}, \mathrm{W}$.

S. 161. Z. 2-3. J: interessanten Angelegenheit

SW: interessanten Gelegenheit $\mathrm{Ku} \mathrm{ScH}, \mathrm{B}, \mathrm{R}, \mathrm{W}$.

S. 161. Z. 10. J: seiner Belehrung (sc bedürfen)

SW: seine Belehrung (sc bedürfen) $\mathrm{KL} \mathrm{ScH}, \mathrm{B}, \mathrm{R}, \mathrm{W}$.

S. 161. Z. 14. J: Nöthige

SW: Nöthigste Ku ScH, B, R, W.

S. 161. Z. 20 . J: gehört in die

$S W$ : gehören in die $\mathrm{Ku} S c H, B, R, W$.

S. 162. Z. 19. J: das Meiste erleichtert wird

SW: das Meiste erreicht wird $\mathrm{KLSCH}, \mathrm{B}, \mathrm{R}, \mathrm{W}$.

S. 162. Z. 32--33. J: eine andere Geistesnahrung bereiten

STV: eine andere bieten $\mathrm{KL}_{\mathrm{L}} \mathrm{SH}, \mathrm{B}, \mathrm{R}, \mathrm{W}$.

S. 162. Z. 35-36. J: den Kopf anfüllen

SW: der Kopf anfüllen $\mathrm{KL}_{\mathrm{L}} \mathrm{ScH}, \mathrm{B}, \mathrm{R}, \mathrm{W}$.

S. 164. Z. 1. J: der feine, glückliche Ausdruck

SW : der freie, glückliche Ausdruck $\mathrm{Ku} \mathrm{ScH}, \mathrm{B}, \mathrm{R}, \mathrm{W}$.

S. 164. Z. 7. J: andre

SW: andere $\mathrm{KL} \mathrm{ScH}, \mathrm{B}, \mathrm{R}, \mathrm{W}$.

S. 164. Z. 21. J: dann, wann

SW: dann, wenn $\mathrm{KL} \mathrm{ScH}, \mathrm{B}, \mathrm{R}, \mathrm{W}$.

S. 164. Z. 31. J: vor allem andern

SW: vor allen andern $\mathrm{Ku} \mathrm{ScH}, \mathrm{B}, \mathrm{R}, \mathrm{W}$.

S. 164. Z. 35. J: bekannt ist, dringt

SW: bekannt, dringt $\mathrm{KL} \mathrm{ScH}_{\mathrm{S}} \mathrm{B}, \mathrm{R}, \mathrm{W}$.

S. 164. Z. 38. J: einnehmen solle

SW: einnehmen soll KL Scr. B, R, W.

S. 165. Z. 7. J: hinwegziehen

SW: hier wegziehn $\mathrm{KL} \mathrm{ScH}, \mathrm{B}, \mathrm{R}, \mathrm{W}$.

S. 165. Z. 12. Mit den Worten: „Ausser diesem" beginnt in SW, sowie in $\mathrm{KL}_{\mathrm{SCH}}, \mathrm{B}, \mathrm{R}, \mathrm{W}$ ein Absatz. In $\mathrm{J}$ dagegen ist fortlaufender Text.

S. 165. Z. 21. J: werden konnte

SW: werden könnte $\mathrm{Ku} \mathrm{ScH}, \mathrm{B}, \mathrm{R}, \mathrm{W}$. 
S. 165. Z. 21 . J: u. dgl.

SW: und dergl. B, W (und dgl. $\mathrm{KL} \mathrm{ScH}, \mathrm{R}$ ).

S. 165. Z. 27--28 J: von diesem, gleichwohl ganz wesentlichen Elementarmittel des Unterrichts

SW: von diesen, gleichwohl ganz wesentlichen Elementarmitteln $\mathrm{KL} \mathrm{ScH}, \mathrm{B}, \mathrm{R}, \mathrm{W}$.

S. 165. Z. 31. J: vollkommener

SW: vollkommner $\mathrm{KLSCH}, \mathrm{B}, \mathrm{R}, \mathrm{W}$.

S. 166. Z. 8. J: voraussetzen: der Boden ist $\mathrm{K} \iota \mathrm{Sch}, \mathrm{R}$

SW: voraussetzen: Boden ist $B, W$.

S. 166. Z. 11-12 J: dem Masse, den Empfindungen

SW: dem Maasse der Empfindungen KLScH, B, R, W.

S. 166. Z. 28. J: angegebnen

SW: angegebenen $\mathrm{KL} \mathrm{ScH,} \mathrm{B,} \mathrm{R,} \mathrm{W.}$

S. 166. Z. 29. J: Charakter jedes Kindes

SW: Charakter des Kindes $\mathrm{Ku} \mathrm{ScH,} \mathrm{B,} \mathrm{R,} \mathrm{W.}$

S. 167. Z. 11, 23, 23-24. J: Etwas anders

SW: Etwas anderes $\mathrm{KL}_{\mathrm{LCH}} \mathrm{Sc}, \mathrm{B}, \mathrm{R}, \mathrm{W}$.

S. 167. Z. 31. J: So sahen

SW: So sehen KL Sch, B, R, W.

S. 168. Z. 1. J: anbieten dürfte

SW: anbieten durfte KL ScH, B, R, W.

S. 168. Z. 5. J: erste (gesperrt)

SW: erste (nicht gesperrt) $\mathrm{KL} \mathrm{ScH,} \mathrm{B,} \mathrm{R,} \mathrm{W.}$

S. 168. Z. 6. J: Festsetzung jener Reihenfolge

SW: Fortsetzung jener Reihenfolge $\mathrm{KLSScH}_{\mathrm{L}} \mathrm{B}, \mathrm{R}, \mathbf{W}$.

Paginirung. Im Texte ist die Paginirung des Textes der Irene, unter dem Texte die entsprechende der Ausgabe SW, KL Scн, B, R, $\mathrm{W}$ angegeben.

\section{VI.}

\section{Pestalozzis Idee eines A B C der Anschauung.}

I. Ausgabe 1802. II. Ausgabe 1804. S. 169--309.

In dem S. 155 citirten Briefe von Halem stellt Herbart eine ,ausführliche Darstellung“" seiner Ansichten über die Pestalozzische Unternehmung in Aussicht. Diese ,ausführliche Darstellung" sollte den Blick „über die nothwendigen Gränzen der Pestalozzischen Absicht" erweitern und die Aufgabe haben, die ,aesthetische Wahrnehmung als den Haupt-Nerven der Erziehung" darzustellen.

An Gries schreibt er im Juli 1802 von Göttingen aus:

„Meine Schriftstellerei wirst Du um Michaelis ganz von unten auf dienen sehen; sie fängt vom A-B-C an. Nämlich von Pestalozzi's Idee 
eines A-B-C der Anschauung, die ich durch und für Mathematik ausgeführt wünsche.: Er fügt hinzu, dass sein Aufsatz in der Irene diese Arbeit, gewissermassen verkündigt".

Die Schrift erschien im Herbst 1802 unter dem Titel:

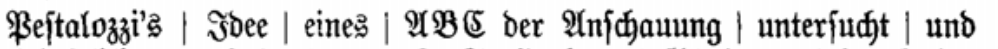

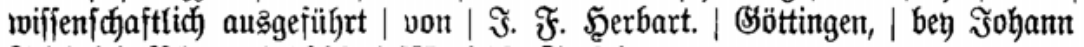

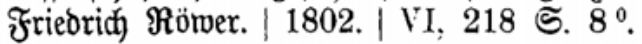

Herbart hatte dieselbe seinem räterlichen Freunde G. A. v. Halem gewidmet. In dem Begleitbriefe, Güttingen, 28. Oct. 1802, schreibt er an Halem: „Wem anders als Ihnen, mein sehr verehrter Freund, könnte ich meine Erstlinge darbringen? Ich habe nicht ror dem Publienm mit Ihnen schwatzen wollen; aber nichts desto weniger steht die ganze Reihe der Jahre vor mir, worin ich die Zeichen Ihrer Aufmerksamkeit, die Ermunterungen Ihrer Grüte nach einander empfing. Sie haben mich zweymal dem Publicum rorgeführt: Sie sind der erste, den ich bei meinem Herrortreten hochachtungsvoll zu begrüssen habe. Meine Schritte werden noch immer langsam seyn. Nur darstellen will ich mich und meine Gedanken der Prüfung. In diesem Geiste werden Sie mein Buch geschrieben, und würden Sie meine hiesigen Verhältnisse, wenn Sie hier wären, eingeleitet sehn ..... Im hohen (rrade würde ich es meiner Vaterstadt verdanken, wenn sie sich das Verdienst um mich erwürbe, die ersten genanen und sorgfältigen Versuche mit meinem Vorschlage anzustellen. - Auf jedem Fall aber darf ich annehmen, dass sie Männer besitzt, die Geist und Interesse genug vereinigen, um sich der grossen Pestalozzischen Idee, elementarische Anschauungen zum Hauptfundament des Unterrichts zu machen, völlig zu bemächtigen. Lnd so darf ich erwarten, von dort aus wenigstens durch Urtheile belehrt zu werden, ob ich jene Idee der Ausführung näher gebracht oder sie verfehlt habe":.

Schon nach zwei Jahren, im Jahr 1804, erschien eine 2. mehrfach umgearbeitete Auflage unter dem Titel:

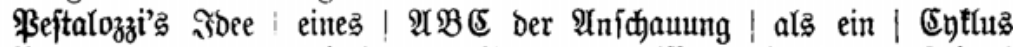

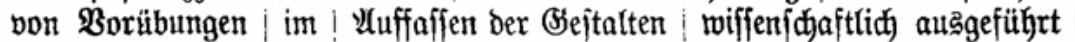

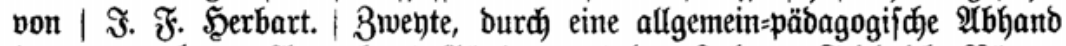

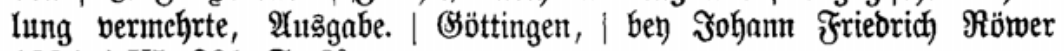
1804. | VI, 281 S. $8^{\circ}$.

Bereits der Titel zeigt die Veränderung, die innerhalb der 2 Jahre seit dem Erscheinen der 1. Auflage in Herbart vorgegangen ist. Im Jahre 1803 war Pestalozzi's ABC der Anschauung oder Anschauungslehre der Massverhältnisse (ron Krüsi bearbeitet) erschienen. Die Ausführung wich sehr von Herbarts Auffassung ab. Hierdurch musste der Zusammenhang seiner Schrift mit Pestalozzi's bezüglichen Bestrebungen gelockert werden. Der Titel der 2. Auflage . . . . „Cyklus von Vorübungen im Auffassen der Gestalten, soll sicher nur den Gegensatz zu dem Nebentitel des Pestalozzischen Buches bezeichnen. Herbart gesteht 
selbst in der Nachschrift zur 2. Auflage: „Wiewohl nun die Verehrung des Verfassers gegen den edlen Schweizer um Nichts gemindert ist; so muss er doch, schon um nicht zudringlich zu seyn, sein Buch aus dem äussern Zusammenhange, worin es stand, um etwas zurückziehn. Die Frage: um wieviel es die Pestalozzischen Plane fördere, kann nicht mehr ein annehmlicher Massstab zu seiner Würdigung seyn. Da es aber zu klein ist, um allein zu stehn, so bleibt ihm nichts übrig, als desto fester an die reine Idee der Pädagogik selbst, auf die es schon vorher vielfältig sich berief, sich anzuschliessen".

Die im Titel der 2. Auflage als Zusatz erwähnte Abhandlung handelt: „Ueber die aesthetische Darstellung der Welt als das Hauptgeschäft der Erziehung".

Herbart bezeichnet diese Abhandlung (S. 288) als ,ein Fragment aus einem älteren Aufsatze, der ursprünglich zur Verständigung mit einem Freunde geschrieben wurde." Dieser Freund ist sehr wahrscheinlich Smidt.

Ausser der „Nachschrift" (S. 283-291) und der Abhandlung: Ueber die aesthetische Darstellung etc. weist die 2. Auflage gegenüber der ersten noch viele Abweichungen auf. Dieselben bestehen in

$$
\begin{aligned}
& \text { Zusätzen, } \\
& \text { Umarbeitungen } \\
& \text { und Weglassungen. }
\end{aligned}
$$

Drucke. Die rorliegende Schrift ist abgedruckt in SW XI, 75233, B II, 79--206, R II, 1-124, W I, 101-224 u. 257-297. SW, B, R legen dabei die II. Ausgabe zu Grunde und notiren die Abweichungen der I. Ausgabe und zwar SW unter, B u. R theils unter, theils am Ende der Sehrift. W legt den Text der I. Ausgabe zu Grunde und giebt die Abweichungen der II. Ausgabe unter dem Texte.

Die angehängte Abhandlung: Ueber die aesthetische Darstellung etc. erschien ausserdem separat noch in $\mathrm{KL} \mathrm{ScH} \mathrm{I,} \mathrm{41-65.}$

Grundlage. Der Text der I. Ausgabe (1802) unter Beifügung aller Abweichungen des Textes der II. Ausgabe (1804). Die Abweichungen sind, um sie als gleichwerthig mit dem Text der Grundlage äusserlich zu kennzeichnen, in den Typen (Corpus) der Grundlage gedruckt. Durchweg sind ferner die Abweichungen der Ausgabe SW von der Originalien notirt. In diesen Fällen der Abweichung ist auch der Text der Ausgaben B, R, W und für die Abhandlung im Anhang der Text ron $\mathrm{KLSCH}$ zur Vergleichung herangezogen und sind die etwaigen Varianten dieser Ausgaben angegeben worden.

Die Abweichungen von SW in erster Linie und die Abweichungen von B, R, W sind ausser durch Typen (Petit) auch noch dadurch in ihrem secundären Verhältnisse $z u$ den Originalausgaben äusserlich gekennzeichnet, dass sie durch einen Strich von den Varianten dieser Ausgaben getrennt sind. 


\section{Textveränderungen.}

S. 178. Z. $22-23$. etwas so bekanntes (II. Ausg) . . . statt . . . eben so bekanntlich.

S. 196. Z. 1. Seile (II. Ausg.) . . . statt . . . Seil.

S. 207. Z. 10. Es ist zwar klar (II. Ausg.) . . . statt . . . Es ist aber klar.

S. 208. Z. 13. unterscheidet danu freylich (II. Ausg.) . . statt . . . unterscheidet denn freylich.

S. 219. Z. 2-3. wird man am besten (II. Ausg.) . . . statt . . . wird am besten.

S. 226 . Z. 39 u. S. 227. Z. 35 . nach gemeinem ... statt nach ... gemeinen.

S. 250. Z. 29. 1771, 65 . . statt . . 1771, 55 .

S. 266. Z. 7. Reihe XI (II) . . statt . . Reibe IX.

S. 271. Z. 17. den Gegendienst . . . statt . . . der Gegendienst.

S. 287. Z. 43. seien . . . statt . . . seyn.

S. 288. Z. 11-12. Hinter einem $A B C$ ! . . statt . . . Hinter einen $A B C$.

S. 301. Z. 11. dass es nicht dureh . . . statt. . . dass es sich nicht. Varianten.

S. 171. Z. 10. I. Ausg.: Grundlinien einer Theorie der Anschauung

II. Ausg.: Grundlinien einer Theorie der Anschauung. Das Dreyeck ist das Element aller Gestalt.

S. 171. Z. 28. I. Ausg.: Schluss

II. Ausg.: „Schluss" fehlt, statt dessen ist noch hinzugefügt: „Nachschrift; und angehängte Abhandlung: über die aesthetische Darstellung der Welt als das Hauptgeschäfft der Erziehung".

S. 176. Z. 2-3. I. Ausg.: selbst einstellen. Scheint

II. Ausg.: selbst einstellen. Vielleicht vermisst man hier die Erwähnung der Massverhältnisse: allein diese liegen in jeder wirklichen Gestalt . . . . o oft die Rede seyn. Scheint.

S.176. Z. 30. \$.177. Z.13. I.Ausg.: Was sie dafür leisten zu können glaubt, wird man im Verfolg finden. Im Voraus verbittet sie nur das Vorurtheil .... von der Rohheit zur künstlerischen Vollkommenheit, hinlänglich klar"

II. Ausg.: hat für diesen Abschnitt folgende Variante (S. 176, 39-177, 40) ,Pestalozzi's Genie gab die Idee ...... bedarf es für die ersten keiner eignen Sorge".

S. 178. Z. 19. I. Ausg.: heisst bey Kindern: ein II. Ausg.: heisst bei den Kindern zunächst: ein.

S. 178. Z. 22-23. I. Ausg.: eben so bekanntlich

II. Ausg.: etwas so bekanntes SW, B, R, W, aber nur $\mathrm{R}$ giebt die Variante von I. 
S. 178. Z. 31. I. Ausg.: zu vergegenwärtigen

II. Ausg.: Z. $34-41$ ) zu vergegenwärtigen. Um aber ron einer geübten Anschauung den ginzen möglichen Gewinn zu ziehen ... davon redet der letzte Abschnitt.

S. 179. Z. 2. I. Ausg.: Die Bildung ... B, R ohne Angabe der Varianten von II

II. Ausg.: Die Ausbildung . . SWW, W ohne Angabe der Varianten von I.

S. 181. Z. 6--15. I. Ausg.: Aber der Vorsatz, der überlegte Entschluss ...... das Beste gethan: so spannt II. Ausg.: hat statt dessen folgende Variante: Aber der blosse Vorsatz, der allgemeine Entschluss ...... das Meiste gewonnen, so spannt .. SW, B, W geben die Variante vou I nicht vollständig; sie lassen die Worte: "das Beste gethan“" weg.

S. 181. Z. 18. I. Ausg.: Das alles, wie viel schlimmer wird es beim Unterricht!

II. Ausg.: Wie viel schlimmer wird dies beym Unterricht! B : ... wird es beym Unterricht!

S.181. Z.25. S.182. Z.11-12. I. Ausg.: . . . muss man es da nicht aufgeben, den Unterricht auf Anschauung zu gründen? .... gethan werden könne?

S.181. Z.29. S.162. Z.39. II. Ausg.: muss man es da nicht aufgeben, den Geist bey dem Sinne zu fassen ..... studirt werden können? W giebt die Variante unvollständig(s.S.182 Anm.).

S. 185. Z. 9. I. Ausg.: vollkommne

U. Ausg.: vollkommene SW, B, R, W ohne Angabe der Variante.

S. 190. Z. 42. I. u. II. Ausg.: diese Grössenbegriffe die Grössenbegriffe SW, W, ohne Angabe der Veränderung.

S. 192. Z. 13. I. Ausg.: an dem ... SW, B, R, W ohne Angabe des Fehlers von II.

II. Ausg.: an den.

S. 192. Z. 14. I. u. II. Ausg.: an so vielem an vielem $\mathrm{SW}, \mathrm{W}$.

S. 194. Z. 4. I. u. II. Ausg.: oder 10ten und 10ten SW, W ohne Angabe der Neränderung. 
S. 194. Z. 37. I. Ausg.: ihr dafür zugesteht

II. Ausg.: ihr dafür zusteht . . SW, B, R, W ohne Ang. der Variante.

S. 196. Z. 1. I. Ausg.: schmalen Seil

II. Ausg.: schmalen Seile . . . sW, B, R, W ohne Angabe der Variante.

S. 197. Z. 20 [19]. I. u. II. Ausg.: und kömmt

und kommt SW, ohne Angabe der Veränderung.

S. 199. Z. 30 -33. I. Ausg.: Daraus muss sich zuerst das Materiale, -daraus müssen sich die Buchstaben ..... . Begriffe zu bringen.

II. Ausg.: Darans muss sich zuerst das Materiale für unsre Vorübungen ergeben .... Begriffe zu bringen.

S. 202. Z. 33. I. Ausg.: zusammengefasst

II. Ausg.: zusammengefasst*

* Um dies, und das zunächst folgende ..... und

für 4 Puncte ein Viereck u. s. f. $(202,36$. $-203,41)$.

S. 203 . Z. 39 [38]. I. Ausg.: sind unzählbar

II. Ausg.: sind unzählig . . SWW, B, R, W ohne Angabe der Variante.

S. 204. Z. 42. I. Ausg.: an dem Original

II. Ausg.: in dem Origina! . . SW, R, B, R, W ohne Angabe der Variante.

S. 204. Z. 41. und S. 205. Z. 1. I. Ausg.: Welches sind denn die... seiner Form?

II. Ausg.: Welches sind denn die .... seiner Form? (gesperrt) (SW, $\mathrm{B}, \mathrm{R}, \mathrm{W}$ ohne Angabe des Unterschiedes von I).

S. 205. Z. 2--3. I. Ausg.: Einfache Puncte

II. Ausg.: Einfache Puncte (gesperrt) ... SW, B, R, W ohne Angabe des Unterschiedes von I.

S. 205. Z. 4. I. Ausg.: sind Nichts für die Form

II. Ausg.: sind Nichts, weder für die Form noch für das Mass. B, aber ohne Angabe der Variante von I.

S. 205. Z. 11. I. Ausg.: mehrere zusammengefasst

II. Ausg.: mehrere zusammengesetzt . . . SW, B, R, W ohne Angabe der Variante von I.

S. 206. Z. 38. I. Ausg.: der Anschauung

II. Ausg.: der Anschauung. Man muss sie ganz und im Zusammenhange verstehn, um in die Meinung der gegenwärtigen Schrift eingehn zu können. 
S. 207. Z. 10. I. Ausg.: Es ist aber klar

II. Ausg.: Es ist zwar klar . . . SW, B, R. W ohne Angabe der Variante ron I.

S. 208. Z. 13. I. Ausg.: unterscheidet denn freylich

II. Ausg.: unterscheidet dann freylich . . . sW, B, R, W ohne Angabe der Variante ron I.

S. 209. Z. 19--20. I. Ausg.: angeregt werden könne

II. Ausg.: angeregt werden kann . . SW, B, R, W; aber nur $\mathrm{R}$ giebt die Variante ron $\mathrm{I}$.

S. 210. Z. 7. I. Ausg.: versuchen

II. Ausg.: versuchen*

* „Schon in der ersten Ausgabe waren die Worte .... Ausschmückung hat gelangen können *(S. 210. Z. $31-45)$.

S. 214. Z. 6. I. Ausg.: metallenen Diese Variante ist im Texte nicht

II. Ausg.: metallnen $\}$ notirt worden.

S. 215. Z. 15. I. Ausg.: vorfinden wird

II. Ausg.: vorfinden wird*

* Dieșe Stelle ist in einer sehr schätzbaren Recension ... der getadelten Stelle (S. 215. Z. 36-43).

S. 219. Z. 2-3. I. Ausg.: "wird an besten

II. Ausg.: wird man am besten. . . . SW, B, R, W ohne Angabe der Variante.

S. 219. Z. 29. I. Ausg.: grade

II. Ausg.: gerade . . . SW, B, R, W ohne Angabe d. Var.

S. 225. Z. 22. I. Ausg.: also statt

II. Ausg.: also anstatt... SW, B, R, W ohne Ang. d. Var.

S. 236. Z. 12-17 [11-16]. I. Ausg.: Die erste, und die zweyte Behauptung ... Beweis statt seiner führen.

II. Ausg.: In der zweiten Ausgabe fehlt der ganze Satz. SW, B, W haben den Satz der I. Ausg. übersehen, dagegen $R$, welche nach II druckt, den Satz als Variante von I notirt.

S. 238. Z. 31. I. u. II. Ausg.: wären irrationale wären rationale SW.

S. 241. Z. 5. I. u. II. Ausg.: Tang. . . . Sec. Tangente ... Secante ... SW, R, W.

S. 243. Z. 21-22. I. u. II. Ausg.: darin liegen 9 Hundertel darin 9 Hundertel ... SW.

S. 243. Z. 23. I. Ausg.: 1,55; zwey ... SW, B, R, W ohne Angabe der Variante von II.

II. Ausg.: 15,5; zwey.

S. 244. Z. 30. I. Ausg.: weil für alle $\ldots$ SW, B, R, W ohne Angabe der Variante von II.

II. Ausg.: weil sie für alle. 
S. 245. Z. 1. I. Ausg.: . . 143=155. W druckt richtig nach I, aber ohne Angabe der Variante von II.

II. Ausg.: $144=155$. . . S SW, B, R drucken den falschen Text ron II, ohne Ang. der Variante ron I.

S. 250. Z. 24. I. Ausg.: $1143 \mathrm{R}, \mathrm{W}$ ohne Ang. der Variante ron II. II. Ausg.: $1134 \mathrm{sW}, \mathrm{B}$ ohne Ang. der Variante ron I.

S. 250 . Z. 29 . I. Ausg.: 154

Il. Ausg.: 1, $04 \mathrm{sW}, \mathrm{B}, \mathrm{R}, \mathrm{W}$ ohne Ang. d. Var. von I.

S. 250 . Z. 31. I. Ausg.: $6246 \mathrm{R}$, W II. Ausg.: $6245 \mathrm{sW}, \mathrm{B}$

S. 250. Z. 33. I. Ausg.: $5115 \mathrm{R}, \mathrm{W}$ SW u. B geben den in correcten Text von II, o hne Angabe der Varianten ron $\mathrm{I}$.

II. Ausg.: $5105 \mathrm{sw}, \mathrm{B}$

S. 250 . Z. 35. I. Ausg.: 527 R, W

II. Ausg.: 517 SiW, B

$\mathrm{R}$ u. W drucken richtig, aber nur $\mathrm{R}$ giebt die Varianten.

S. 252. Z. 1. I. Ausg.: $\frac{214.100}{i 73} \ldots \mathrm{B}, \mathrm{R}, \mathrm{W}$ (correct) ohne Angabe der Variante von II.

II. Ausg.: $\frac{214.100}{100} \ldots$ SW (incorrect) ohne Angabe der Var. von $\mathrm{I}$.

S. 254. Z. 36. I. Ausg.: Daraus zerstreut liegen ${ }^{1}$

II. Ausg.: Durchaus zerstreut .... S SW, B, R, W ohne Angabe der Varianten ron I.

S. 255. Z. 35-36. I. u. II. Ausg.: angeben wollte abgeben wollte $\mathrm{SW}$.

S. 256. Z. 35. I. Ausg.: 1,08 S'W, B, R, IV (correct) ohne Angabe der Varianten ron II

II. Ausg.: 10,8 .

S. 256. Z. 39. I. Ausg.: musste (gesperrt)

II. Ausg.: musste (nicht gesperrt) SW, B, R, W ohne Angabe der Variante.

S. 257. Z. 32 . I. u. II. Ausg.: Das bisherige Die Bisherige SW.

S. 260. Z. 1. I. Ausg.: ihrem eigensten Wesen

II. Ausg.: ihrem Wesen, ... SW, B, R, W ohne Angabe der Varianten von I.

S. 260. Z. 1-2. I. Ausg.: Begriff erschöpfend ausgedrückt

II. Ausg.: Begriff gehörig ausgedrückt ... SW, B, R, W whe Angabe der Variante von I.

${ }^{1}$ Durch Vergleichung mehrerer Exemplare der I. Ausgabe hat sich herausgestellt, dass nicht: „Daraus“ sondern „Duraus“ (für Durchaus) gedruckt ist. In meinem Handexemplare war das "„1" so undeutlich gedruckt, dass man es für „a“ halten musste. 
S. 260. Z. 2-3. I. Ausg.: Grösse, - das blosse Verhältniss der Form, - ist

II. Ausg.: Grösse ist ... SW, B, W' ohne Angabe der Variante; $\mathrm{R}$ giebt die Variante.

S. 260. Z. 4. I. Ausg.: Zahlbegriff erreicht es wahr und ganz

II. Ausg.: Zahlbegriff erreicht die Verhältnisse der Form* SW, B, W ohne Angabe der Variante; $\mathbf{R}$ giebt die Variante.

S. 260. Z. 34. Die II. Ausgabe hat nach .....Form" eine Anmerkung Form*.

Doch erreicht er .... nicht verfehlen (Z. 35-38).

S. 263. Z. 6. I. Ausg.: sieht man sieht man

II. Ausg.: sieht man ... SW, B, R, W (correct) ohne Angabe des Druckfehlers ron I.

S. 263. Z. 31. I. u. II. Ausg.: als alle andre Columnen als andre Columnen SW, W.

S. 264. Z. 25 [24]. „dienen“ in I. 2 mal gedruckt. Die übrigen Ausgaben drucken correct.

S. 264. Z: $32-33$ [31-32]. I. Ausg.: seyn. Dann ist die Aufgabe: die Winkel zu finden. Desgleichen II. Ausg.: seyn. Desgleicheu ... SW, B, $\mathrm{R}$, W ohne Ang. der Variante.

S. 266. Z. 7. I. Ausg.: Reihe IX

II. Ausg.: Reihe XI . . SW, B, R, W ohne Angabe der Variante von I.

S. 267. Z. 14 [5]. I. u. II. Ausg.: aufgelöseter aufgelöster SW.

S. 268. Z. 3 [S. 267. Z. 13]. I. u. II. Ausg.: darum soviel eher ditrum um soviel eher SW.

S. 269. Z. 21. I. Ausg.: jene Grundformen

II. Ausg.: jene dürftigen Grundformen . . . SW, B, W ohne Angabe der Varianten; $\mathrm{R}$ giebt die Var.

S. 269. Z. 21-22. I. Ausg.: allein, in ihrer armen Einfalt zur Verbesserung

II. Ausg.: allein, zur Verbesserung . . . sW, B, W obne Angabe der Var.; $\mathrm{R}$ giebt die Var.

S. 271. S. 32. I. u. II. Ausg.: Spielerey auch könnte

Spielerey doch könnte... sW ohne Ang. des Originaltextes.

S. 275. Z. 9. I. Ausg.: er enthält ... B, W ohne Angabe der Var. II. Ausg.: er erhält ... S SW, $\mathrm{R}$ ohne Angabe der Var.

S. 279. Z. 34. I. Ausg.: wieder emporsteigend

II. Ausg.: wieder hervorsteigend ... SW, B, R, W ohne Angabe der Variante. 
S. 280. Z. 36. I. u. II. Ausg.: ähnlichen Vorbereitung nämlich ähnlichen Grundlage nämlich . . . SW,

S. 281. Z. 40. I. Ausg.: Studien?

W ohne Angabe des Originaltextes.

II. Ausg.: Seiten ... SW, B. R, W ohne Ang. der Var.

S. 281. Z. 1. 4. 8. 15. I. u. II. Ausg.: frägt

fragt ... SW, W ohne Angabe des Originaltextes.

S. 282. Z. 24-29. I. Ausg.: Das A BC' der Anschauung . . . Zurückbehaltene nachgesendet werden

II. Ausg.: In der 2. Ausg. fehlt dieser Abschnitt.

S. 282. Z. 25. I. Ausg.: ist nur noch ein

ist noch ein ... SW, B, W ohne Angabe des Original-Wortlautes

S. 282. Z. 26. I. Ausg.: Händen, sich auf seine

Händen, auf sein ... SW, B, W ohne Angabe des Original-Wortlautes.

S. 282. Z. 28--29. I. Ausg.: Zurückbehaltne

Zurückbehaltene ... SW, B, R, W ohne

Angabe des Original-Wortlautes.

S. 287. Z. 43. II. Ausg.: seyn

seien ... B, R, W (sein SW), ohne Angabe des Original-Wortlautes.

S. 290. Z. 34. II. Ausg.: synthesischen synthetischen ... SW, B, R, W ohne Angabe des Druckfehlers im Original.

S. 295. Z. $22-23$. II. Ausg.: Erhehung

Erhebung ... SW, B, R, W ohue An-

S. 296. S. 39. II. Ausg.: $\dot{\eta} \delta$ ov $\eta$

gabe des Druckfehlers im Original.

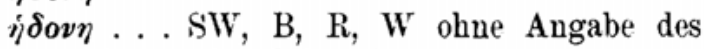
Druckfehlers im Original.

S. 299. Z. 23. II. Ausg.: als einzigen Spruches

als einen Spruches SW.

S. 300. Z. 26. II. Ausg.: im einen Fall

in einem Fall SW.

S. 300. Z. 41 [40]. II. Ausg.: längst gestanden hätte längst gefunden hätte SW.

S. 301. Z. 11 [12]. II. Ausg.: dass es sich nicht dass es nicht SW, K七 Sch, B, R, W ohne Angabe des Original-Wortlautes.

S. 304. Z. 22. II. Ausg.: rückkehrenden zurückkehrenden SW, W.

Paginirung. Im Texte ist die Paginirung des Grundtextes angegeben, also bis S. 282 diejenige der I. Ausgabe, von S. 281 an, wo 
die „Nachschrift zur zweyten Auflage“" beginnt, diejenige der II. Ausgabe. Unter dem Texte ist die Paginirung der Ausgaben SW, B, R, W angegeben; dazu tritt noch für die angehängte Abhandlung: Ueber die aesthetische Darstellung etc. S. 292-309 die Paginirung von KuSch.

\section{VII.}

\section{Thesen zar Promotion und Habilitation [1802]. S. 313-316.}

Nach damaliger Sitte genügte an der Universität Göttingen zur Promotion und Habilitation ein mündliches Examen nebst Disputation. Eine Dissertation wurde weder für die Promotion noch für die Habilitation verlangt.

Ueber den Werth der Thesen urtheilt Hartenstein (SW XII, S. XI): „Jeder der Sätze, die sie (sc. die Thesen) enthalten, ist der Ausdruck eines in seiner Sphäre zur Reife gediehenen Denkens; keinen derselben hat Herbart später zurückzunehmen sich veranlasst gefunden, und mit ihnen kann die Periode der Vorbereitung als abgeschlossen angesehen werden. Sie zeigen, dass, die Principien der Ethik ausgenommen, er damals schon über das Verhältniss der verschiedenen Gebiete der philosophischen Untersuchung sammt den Grundgedanken der Metaphysik und Psychologie mit sich ins Reine gekommen war." R. Zimmermann ist derselben Meinung. „Mit den Thesen ist Herbart's philosophische Wanderzeit zu Ende; sein Geist tritt in die Meisterjahre ein." Sitzungsberichte der [Wiener]Akademie der Wissenschaften, (philos.-histor. Classe) Bd. 83, Jahrg. 1876. S. 225 и. 226.

Manuscript. Das Manuscript der Thesen befindet sich in den Dekanats-Acten der philos. Facultät zu Güttingen Fasc. 86 (1802-1803).

Drucke. Die Thesen sind rollständig in SW XII, S. 58 и. 59.

u. KLSCH I, LVIII und LIX abgedruckt.

Grundlage. Das Manuscript.

Varianten :

S. 315. Z. 9. Msc.: philosophia, totum (Komma)

SW: philosophia totum (ohne Komma) KuScH.

S. 315. Z. 12. Msc.: Sed, si (Komma)

SW: Sed si (ohne Komma) KuScн.

S. 315. Z. 13. Msc.: nec plene

SW: nec plane KLScH.

S. 316. Z. 9. Msc.: agnitio, animique (Komma)

SW: agnitio animique (ohne Komma) KLScH.

S. 316. Z. 11. Isc.: rursus exoritur

SW: rursum exoritur (KLSCH). 
Ausserdem rariiren SW u. KLSCH noch dadurch rom Msc., dass sie einige der lateinischen Substantiva, welche Herbart mit grossen Anfangsbuchstaben schreibt, mit kleinen Buchstaben drucken.

Paginirung. Unter dem Texte ist die entsprechende Paginirung von S'W u. KLSCH angegeben.

\section{VIII. \\ Zwei Vorlesungen über Pädagogik [1802].}

Diese Vorlesungen sind die ersten, welche Herbart in seinem Colleg über Pädagogik im ersten Semester (1802) seiner Wirksamkeit gehalten hat. Für das erste Semester hatte er angekündigt: „Pädagogik nach Dictaten mit Beifügung einer besondern Unterhaltungsstunde." Hartenstein lässt in KLSch I, S. LXIv dahin gestellt, ol, das Jahr 1802 ,ganz genau ist". Dass die Reden aber ,in die erste Zeit seiner akademischen Wirksamkeit fallen", ist ihm gewiss. Ebenso erklärt er SIV XI, S. vII, dass sie jedenfalls ,in die allererste Zeit seiner akademiscben Thätigkeit" zu setzen seien, vielleicht in das Jahr 1802. In dem chronologischen Verzeichniss der Werke Herbarts SW XII, S. 785 sind die Vorlesungen in das Jahr 1802, dagegen SW XI. S. 61 auf dem Titelblatte in das Jahr 1803 gesetzt worden. B, R, W stellen die Vorlesungen unter das Jahr 1802.

Herbart las erst wieder Pädagogik im Wintersemester 1803/1804. ('egen ein Verlegen der Yorlesungen in dieses Jahr ist geltend zu machen, dass Herbart (S. 322) die Kenntniss seiner Habilitationsthesen voraussetzt, eine Voraussetzung, welche er $1 \mathrm{Jahr}$ später schwerlich mehr machen durfte; er erwähnt ferner (S. 327), dass er „Psychologie und Moral" nicht voraussetzen könne. Im Wintersemester 1803/1804 aber konnte er Moral voritussetzen, denn im Sommersemester 1803 hatte er: „praktische Philosophie oder Horal etc." gelesen. Diese Gründe führt auch Willmamn an. Dazu tritt aber noch ein dringenderer. S. 328 zeigt Herbart (dies fehlt in allen übrigen Ausgaben) seinen Zubörern an, dass er, um Collision mit Dr. Winkelmann zu vermeiden, seine Vorlesungen rerlegen werde. Eine solche Collision konnte nur im Wintersemester 1802/1803 stattfinden, da Winkelmann bereits 1803 die Universität Güttingen verliess und nach Braunschweig übersiedelte. Vielleicht hätte auch die Angabe der Wohnung S. 328, Z. 27 einen weiteren Anhaltepunkt geben können, allein trotz aller Recherchen in Göttingen gelang es nicht, zu erfahren, wo Herbart in dem Jahre 1802 gewohnt und wie der im Msc. undeutlich geschriebene Name des Vermiethers gelautet habe. 
Manuscript. Das Msc., welches leider die 2. Vorlesung nur im Fragment enthält, befindet sich auf der Königsberger Universitätsbibliothek. Es besteht aus $12 \mathrm{Bl}$. Von $\mathrm{Bl}$. 10 ist die Rückseite nicht beschrieben, mit Bl. 11 beginnt die 2. Rede. Es enthält vielfach Spuren von Hartensteins redactioneller Thätigkeit. Viele Ergänzungen der Herbart'schen Abkürzungen, die für den Setzer gültigen Bemerkungen am Rande rühren ron Hartenstein her. Den Anfang der Vorlesung hat Herbart stilistisch nicht ausgearbeitet. Unter der Ueberschrift „Pädagogik" folgen als erste Zeile die Stichworte: „Keine Definition, - Lobrede, - Geschichte, - Ueberblick". Am oberen rechten Rande der Handschrift hat Herbart angefangen, die Stichworte in Sätze aufzulösen; er hat dies jedoch nur mit dem ersten Worte gethan. Der Satz lautet: „An der Spitze dieser Vorlesungen erwarten Sie, m. H., vielleicht vor allem andern die Definition meines Gegenstandes, erwarten sodann ...." Das Msc. schliesst mit den Worten: „Zwey Eigen..."

Druck. Die Vorlesungen sind abgedruckt in SW XI, S. 61-74, KLSch I, S. 1-16, B II, S. 67-78, R II, S. 269-280, W I, S. 224-242 unter dem Titel: Rede bei Eröffnung der Vorlesungen über Pädagogik; W hat den Zusatz: „nebst einem Bruchstück aus der zweiten Vorlesung". Ganz vollständig ist das vorhandene Msc. bis jetzt noch nicht abgedruckt worden. Die Ausgaben $\mathrm{B}, \mathrm{R}, \mathrm{W}$ geben den gekürzten Text von SW u. KuScH. Die Kürzungen s. Varianten.

Grundlage. Das Manuscript.

Textveränderungen. Die erwähnten Stichworte sind in einen vollständigen Satz umgewandelt worden: „An der Spitze dieser Vorlesungen erwarten $\mathrm{Sie}, \mathrm{m}$. H., vielleicht vor allem Andern die Definition meines Gegenstandes, erwarten sodann eine Lobrede, eine Geschichte, einen Ueberblick desselben“. S. 323,16 zum wenigsten statt ... zum wenigstens.

\section{Varianten.}

S. 320. Z. 9. Msc.: gewiss der Pädagogik

SW: gewiss für die Pädagogik $\mathrm{KLSch}, \mathrm{B}, \mathrm{R}, \mathrm{W}$.

S. 320. Z. 29. Msc.: sehn

SW: sehen $\mathrm{K}_{\mathrm{LSCH}}, \mathrm{B}, \mathrm{R}, \mathrm{W}$.

S. 321. Z. 5. Msc.: ron dem Einfluss

SW: von dem Einflusse $\mathrm{KrSch}, \mathrm{B}, \mathrm{R}, \mathrm{W}$.

S. 322. Z. 35. Msc.: Unsre

SW: Unsere $\mathrm{KrSch}, \mathrm{B}, \mathrm{R}, \mathrm{W}$.

S. 323. Z. 16. Msc.: zum wenigstens

SW: zum wenigsten $\mathrm{KLSCH}_{\mathrm{L}} \mathrm{B}, \mathrm{R}, \mathrm{W}$.

S. 323. Z. 19. Msc.: geschehn

$\mathrm{SW}$ : geschehen $\mathrm{KLScH}, \mathrm{B}, \mathrm{R}, \mathrm{W}$.

S. 323. Z. 20. Msc.: er gehabt hätte

$\mathrm{SW}$ : es gehabt hätte $\mathrm{KLSCH}, \mathrm{B}, \mathrm{R}$. 
S. 323. Z. 21-22. Iss.: ihm rielleicht nur nicht SW: ihm nur nicht $\mathrm{KLSSH}_{\mathrm{CH}}, \mathrm{B}, \mathrm{R}$.

S. 323. Z. 31. Msc.: ahnden

SW: ahnen $B$.

S. 323. Z. 36. Msc.: andre

$S W$ : andere $\mathrm{KLSCH}_{\mathrm{L}}, \mathrm{B}, \mathrm{R}$.

S. 325. Z. 2. IIsc.: restsetzen werde?

SW: restsetzen (resp. festsetzen) wird? $B, R, W$.

S. 325 . Z. 6 u. 10. Nsc.: unsre

SW: unsere lísich, B, R, W.

S. 325. Z. 13. Msc.: unsrer

SW: unserer Kísch, B, R. W.

S. 326. Z. 20. Hsc.: mancher Anregung

SW: manchen duregungen lís SH, B, R, W.

S. 326. Z. 23. IIsc.: nur Anfangs

SW: nun Anfangs $\mathrm{KLS}_{\mathrm{CH}}, \mathrm{B}, \mathrm{R}, \mathrm{W}$.

S. 327. Z. 27-28 des Msc. hat einen Absatz, ebenso W; SW, B, R haben dagegen keinen Absatz.

S. 328. Z. 17-30. Isc.: Bey jedem Vortrage, der sich dem philosophischen nähert .... Freỹtags um diese Stunde lesen werde.

In SW sowie atich in $\mathrm{KLSCH}_{\mathrm{L}}, \mathrm{B}, \mathrm{R}, \mathrm{W}$ fehlt dieser Schlusssatz.

S. 330. Z. 25- 34. Msc.: Lassen Sie uns jetzt den Begriff .... Zwey Eigen .... .

In SW sowie auch in $\mathrm{KLS} \mathrm{SCH}_{1}, \mathrm{~B}, \mathrm{R}, \mathrm{W}$ fehlt dieser Abschnitt.

S. 323. Z. 19--20. Der Satz: „wemn er anders gehandelt, welchen Erfolg er gehabt bätte", fehlt in W.

Paginirung. Im Texte ist die entsprechendePaginirung des Manu scriptes angegeben, unter dem Texte diejenige ron SW, KLSCr, B, R, u. W.

\section{IX.}

\section{Kurze Darstellung eines Plans zu philosophischen Vorlesungen 1804. S. 331-340.}

In der Vorrede zur 2. Auflage des Lehrbuchs zur Einleitung in die Philosophie giebt Herbart den Beweggrund für die Abfassung vorliegender Schrift an: „Als ich in Göttingen anfing, philosophische Vorträge zu halten, wurde mir der wohlwollende Rath ertheilt: was auch mein Collegium sein möge, Logik und Metaphysik müsse es heissen, 
um in Gang zu kommen. Für diese Zusammenstellung des Leichtesten und des Schwersten hatte und habe ich keinen Sinn; beinahe ebenso gut könnte man ein Collegium über Regel de tri und Integralrechnung ankündigen. Darum entwarf ich im Jahre 1804 den Plan zu einer Einleitung in die Philosophie, welche das Selbstdenken der Anfänger möglichst vollständig $z u$ den Problemen hinführen sollte; und wobei ich die Winke benutzte, welche über den natürlichsten Gang des Denkens die ältere Geschichte der griechischen Philosophie darbietet.“

Titel:

Kurze | Darstellung eines Plans : zu | philosophischen Vorlesungen. | Von ! Johann Friedrich Herbart. ! Göttingen, gedruckt bey Johann Friedrich Röwer. $1804 . \mid 23 \mathrm{~S} .8^{\circ}$.

Diese Original-Ausgabe $(0)$ ist sehr selten und wahrscheinlich nur in einer sehr kleinen Auflage gedruckt worden. Hartenstein erwähnt ausdrüicklich in $\mathrm{KLScH} \mathrm{I}$, cxv, dass sie ,wohl den meisten" von Herbarts Freunden ganz unbekannt sei. In Voigdts Verzeichniss der Herbart'schen Schriften fehlt sie. Erst durch eine Anzeige in den Göttinger gelehrten Anzeigen ist Hartenstein auf dieselbe aufmerksam geworden.

Drucke. Abgedruckt ist die Schrift in SW I, 361-371, KLSCH I, 17--28, W I, 243-255.

Grundlage. Die Original-Ausgabe (0), Göttingen 1804.

Varianten. S. 338. Z. 27. 0: zwisehen seiner $\mathrm{KLSCH}_{\mathrm{L}}$

SW: zwischen einer W.

S. 333. Z. 8. 0: Ahndung KLScH

SW: Ahnung W.

S. 339. Z. 26. 0: Un-Verständigste

$s W$ : Unvernünftige $\mathrm{KLSCH}, \mathrm{W}$.

S. 340. Z. 7. 0 : sobald man nur die $\mathrm{K}_{\mathrm{L}} \mathrm{ScH}$

SW: sobald man die W.

Paginirnng. Im Texte ist die Paginirung des Originals (0), unter dem Texte diejenige von SW, KLScH, W angegeben.

\section{X.}

\section{Ueber den Standpunkt der Beurtheilung der Pestalozzischen Unterrichtsmethode. S. 341-350.}

Eine Gastvorlesung gehalten im Museum zu Bremen 1804.

Dass Herbart schon während seines Bremer Aufenthalts sich viel mit Pestalozzi beschäftigte; dass er in Bremen das Interesse für die Pestalozzischen Bestrebungen zu wecken und zu erhalten suchte, ist 
schon erwïhnt (s. S. xvir). Smidt war bei seiner Reform des Bremer Schulwesens auf die Einführung der Pestalozzischen Methode bedacht. Ein Zögling des Bremer Waisenhauses, Blendermann, war zu Pestalozzi geschickt worden, um nach seiner Rückkehr die Einführung dieser Methode in der Elementarclasse des Gymnasiums zu bewirken. Aus einem undatirten Briefe Herbarts an Smidt aus dem Jahre 1802 geht hervor, dass Herbart im Auftrage Smidts an Pestalozzi geschrieben und ibn um Empfehlung eines geeigneten Lehrers für Bremen gebeten hat.

Der obige Vortrag ist im Jahre 1804 gehalten worden. Noch in demselben Jahre erschien derselbe als Brochüre. Titel:

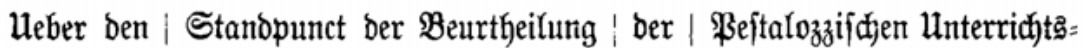
methode / eine Bajtborlejung gehalten im Mujeum zu Bremen | von

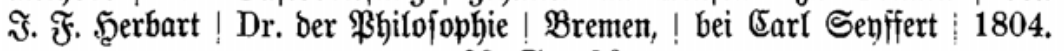
23 ธ. $8^{\circ}$.

Dracke. Der Vortrag ist gedruckt in SW XI, 343-354. KuSCH I, 29-40. B II, 207--216. R II, 259-268. W I, 299-311.

Grundlage: Der Originaldruck (0).

Textveränderungen :

S. 343. Z. 26. dass sie sich aus ... statt . . dase sie aus

S. 344. Z. 42 . seyen ... statt . . . seyn

\section{Varianten :}

S. 343. Z. 26. 0: dass sie aus

SW: dass sie sich aus $\mathrm{KLSCH}_{\mathrm{L}} \mathrm{B}, \mathrm{R}, \mathrm{W}$.

S. 344. Z. 42. 0: seyn (SW: sein) seyen (resp. seien) $\mathrm{KLSCH}_{\mathrm{L}}, \mathrm{B}, \mathrm{R}, \mathrm{W}$.

S. 346. Z. 8. 0: gemachet $\mathrm{SW}$ : gemacht $\mathrm{KuScH}, \mathrm{B}, \mathrm{R}, \mathrm{W}$.

S. 347. Z. 5. 0: in sich selbst die

SW: in sich die $\mathrm{KLSCH}_{\mathrm{L}}, \mathrm{B}, \mathrm{R}, \mathrm{W}$.

S. 347. Z. 28. 0: immer anders und anders baut $\mathrm{KLSCH}, \mathrm{R}, \mathrm{W}$.

SW: immer anders baut $\mathrm{B}$.

S. 348. Z. 13. 0: solle? (Fragezeichen), KLScH, R.

SW: solle, (Komma) B.

(W hat ein Semikolon.)

S. 350. Z. 19. 0: vom Basedow.

SW: von Basedow $\mathrm{KuScH}, \mathrm{B}, \mathrm{R}, \mathrm{W}$.

Paginirung. Im Texte ist die entsprechende Paginirung des Originaldruckes, unter dem Texte diejenige von $\mathrm{SW}, \mathrm{KuScH}, \mathrm{B}, \mathrm{R}$, $\mathrm{W}$ angegeben. 
XI.

De Platonici systematis fundamento commentatio. 1805. S. $351-375$.

Dazu:

Anhang 1: Herbarts Selbstanzeige der vorliegenden Abhandlang. S. $376-377$.

" 2: Böckhs Recension der vorliegenden Abhandlung. S. 378 -387 .

" 3: Herbarts Replik gegen Böckhs Recension. S. 388-394.

Am 28. März 1805 wurde von der Hannöverschen Regierung der Universität Göttingen mitgetheilt, dass Herbart „wegen angerühmten guten Lehrgaben und Talente" ${ }^{1}$ zum Extraordinarius ernannt worden sei. Diese Ernennung war Herbart bereits am 14. Febr. 1805 ,in Rücksicht seiner bekannten Lehrgaben und Talente" unter der Bedingung, dass er den Ruf nach Heidelberg ablehne, in Aussicht gestellt worden.

Als Einladungsschrift zu der üblichen Antrittsvorlesung verfasste er die obige Abhandlung, die nebst dem deutschen Anhange, der in der Finladungsschrift fehlte, im Buchhandel erschien. Der genaue Titel dieser Abhandlung lautet:

De | Platonici systematis | fundamento | commentatio. | Professoris Philosophiae Extraordinarii | in | Academia feorgia Augusta | muneris | rite adeundi gratia | conscripta | auctore | Joanne Friderico Herbart. | Gottingae, | typis Roeverianis. | $1805 . \mid 63 \mathrm{~S} .8^{\circ}$.

Als Anhang und zugleich als Erläuterung der vorliegenden $\mathrm{Ab}$ handlung sind Herbarts Selbstanzeige derselben, die Böckh'sche Recension und die von Herbart gegen die letztere verfasste Replik beigefügt worden.

Die „Selbstanzeige" erschien erst im Jahre 1806 in den Göttingischen gelehrten Anzeigen (G. g. A.) Nr. 76.

Die Recension Böckh's, deren Verfasser in SW XII, S. x山I nicht genannt wird und wahrscheinlich Hartenstein auch nicht bekannt war, ${ }^{2}$ erschien im Jahrgang 1808 der Jenaischen Allgemeinen Literatur-Zeitung $(\mathrm{JL})$ Nr. 224 und 225.

${ }^{1}$ Nach den Curatorial-Acten von Göttingen. Einige der Actenstücke werden zum Abdrucke gelangen.

${ }^{2}$ Die Autorschaft Böckhs ist nach der Ausgabe von Böckhs gesammelten kleineren Schriften Bd. VII, S. 80-92 herausgegeben von F. Ascherson und Paul Eichholz, Leipzig, Teubner, 1872, unzweifelhaft. 
Die Replik erschien darauf in demselben Jahre im Intelligenzblatt der Nenen Leipziger Literatur-Zeitung (LLZ) Nr. 43.

Die strenge Zugehörigkeit zur obigen Abhandlung ist der Grund einer Abweichung ron der chronologisehen Anordnung.

Drucke. Die Abhandlmg De Platonici etc. ist abgedruckt in SW XII, 63-88 und KLSch I, 69-97.

Die ,Selbstanzeige" ist in SIV XII, xI-XII; die Böckh'sche Recension in Böckhs Kleineren Schriften, Bd. VII, S. 80-92, Leipzig, Teubner, 1872; die Replik in SW XII, 88-96 nachgedruckt worden.

Grundlage. Dem Text der Abhandlung: De Platonici etc. liegt der Originaltext, Gottingae 1805, zu Grunde.

Für Anhang 1, 2 und 3 liegen die entsprechenden Originaltexte von G.g. A. Nr. 76, JL Nr. 224 und 225, LLZ Nr. 43 zu Grunde.

\section{Textveränderungen :}

S. 356. Z. 31. cum iurene ... statt cum invene

8. 357. Z. 18. $\tau \epsilon^{\prime} v \tau \kappa$. . statt . . $\tau \epsilon v \tau \kappa$

S. 357. Z. 22. $\alpha v \tau \varepsilon,-($ Komma) . statt . avts - (ohne Komma)

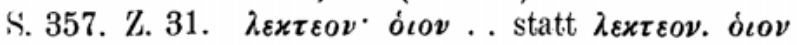

s. 358. Z. 26 . $\varepsilon \sigma \chi \varepsilon v$. . statt $\varepsilon \chi \varepsilon v$

S. 360. Z. 23. $\varepsilon \pi \iota \sigma \times \varepsilon \psi \varphi \iota \nu$. . statt $\varepsilon \pi \iota \sigma x \varepsilon \pi \psi \iota \nu$

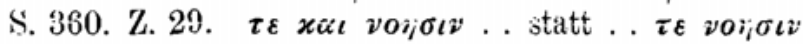

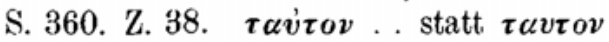

S. 361. Z. 7. TR $T \Omega E E$ statt T $T \Omega \Delta E$

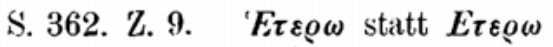

S. 362. Z. 30. $\tau \alpha \dot{i} \tau \alpha$. . statt $\tau \alpha v \tau \alpha$

S. 363. Z. 32 . illud Unum .. statt .. illum Unum

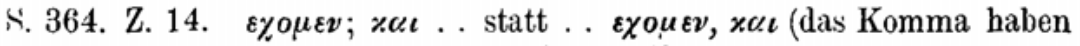
auch SW, KLS'H, Steph., Bip.; nur Bekk. hat Fragezeichen).

S. 364. Z. 17. $\delta \eta \pi \varepsilon \varrho \iota$ statt $\delta \eta, \pi \varepsilon \varrho \iota$ (der Platonische Text lautet: $\delta \eta$, $I I \alpha \rho \mu \varepsilon v \iota \delta \eta, \pi \varepsilon \rho \iota, \boldsymbol{x} . \tau$. $\lambda$.) KLScH hat das Komma beibehalten, SW dasselbe aber getilgt.

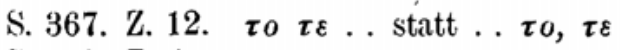

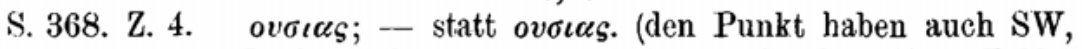
KLScH; das Fragezeichen haben Steph., Bip., Bekk.) S. 373. Z. 10. .. Gleiche!") statt Gleiche!)

Zu Anhang 3. Replik Herbarts in der Leipziger LiteraturZeitung (LLZ).

S. 391. Z. 26. zu offenbarendem) .. statt $z u$ offenbarenden)

S. 392. Z. 23-24. $\delta \iota \iota \sigma \chi v \varrho \iota \sigma \iota \iota \mu \eta \nu$ statt $\delta \varepsilon \iota \sigma \chi v \varrho \iota \sigma \iota \alpha \iota \mu \eta \nu$

Den Platonischen Text citirt Herbart nach der Bipontiner PlatoAusgabe. Nach dem Beispiele Hartensteins sind im vorliegenden Drucke bei allen Citaten die entsprechenden Stellen der Plato-Ausgabe von H. Stephanus hinzugefügt worden. 
Bei den Varianten, welche den platonischen Text betreffen, ist ausserdem die Plato-Ausgabe von Immanuel Bekker herangezogen worden.

Wenn die Citate Rede und Gegenrede enthalten, so sind der Deutlichkeit halber die Worte des Gegenredners in : . . . : eingeschlossen worden.

Varianten. Hartenstein hat in SW und KLScH den griechischen Text der vorliegenden Abhandlung accentuirt (freilich oft fehlerhaft), ebenso hat er in den instrumentalen Bildungen ( $\boldsymbol{\pi} \boldsymbol{\tau} \boldsymbol{\tau} \boldsymbol{\eta} \eta$, ovd $\alpha \mu \eta$ etc.) das Jota subscr. gesetzt, wo Herbart weder accentuirt noch subscribirt. Die hierauf bezüglichen Varianten sind im vorliegenden Drucke, der genau dem Originale folgt und nur über die von ó cuv่rós vorkommenden Formen das Zeichen der Krasis setzt. nicht angemerkt worden.

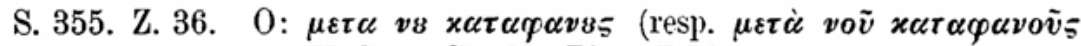
KLScH, Steph., Bip., Bekk.)

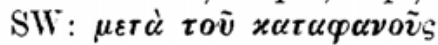

S. 356. Z. 28. 0: quid? quod $\mathrm{KLSCH}$

SW: quid, quod

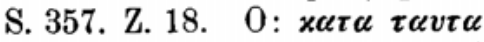

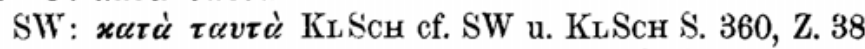

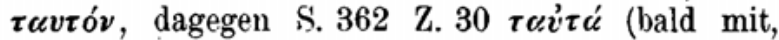
bald ohne Koronis)

S. 357. Z. 34. 0: ad eam KL ScH

SW: at eam

S. 358. Z. 32. 0: dialogicae elegantiae

SW: dialogicae degantiae

S. 359. Z. 21 . 0: diligentia, certi

SW: diligentia certi

S. 361. Z. 29-30. $0: T \iota \delta \varepsilon \varepsilon \varsigma \iota \nu$ ó, $\tau \iota$

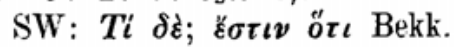

S. 361. Z. 33. 0: $\pi \alpha \nu \tau \alpha, \tau \omega$ (resp. $\pi \alpha^{\prime} \nu \tau \alpha, \tau \tilde{\omega} \mathrm{KL} \mathrm{S} c \mathrm{H}$, Steph., Bip.) $\mathrm{SW}: \pi \tilde{\nu} \nu \tau \alpha \tau \tilde{\omega}$

S. 361. Z. 39. 0: reiicienda, sint (sic)

$\mathrm{SW}$ : reiicienda sint $\mathrm{K}_{\mathbf{L S C H}}$

S. 362. Z. 12. $0: x \alpha \lambda \alpha, \varepsilon x \varepsilon \imath \nu 8 \mathrm{KLSCH}$.

SW: xuोc̀ éxzivov (kein Komma)

S. 362. Z. 30. 0: $\tau$ avt $\alpha$

SW: $\tau$ cuv่ $\dot{c}^{\prime} \mathrm{KLSCH}$ (Akut)

S. 363. Z. 32. 0: illum Unum (s. Textveränderungen)

$\mathrm{SW}$ : illud Unum $\mathrm{KLSCH}$

S. 367. Z. 12. 0: $\tau$ o, $\tau \varepsilon$ (nach Steph., Bip.) (s. Textveränderungen)

$\mathrm{SW}: \tau \dot{o}$ (sic) $\boldsymbol{\tau} \varepsilon$ ( $\boldsymbol{\tau} \dot{o} \boldsymbol{\tau} \varepsilon$ Bekk.)

S. 367. Z. $23.0: \alpha \lambda \lambda \eta \lambda \omega v$, ixo

SW: $\dot{\alpha} \lambda \lambda \dot{\eta} \lambda \omega \nu$ vं $\boldsymbol{x} \dot{o}$ (ohne Komma) Bekk. 


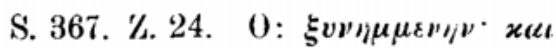

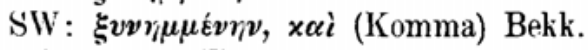

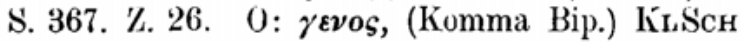

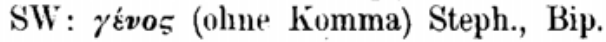

S. 373. Z. 11. 0: Verschiedne

SW: Verschiedene

Zu Anhang 1. Herbarts Selbstanzeige in G.g.A.

S. 376. Z. 7. G. g. A.: Úrheber selbst

SW: Urheber derselben selbst

S. 376. Z. 12. G. g. G.: Philosophen. Beyspiele sind häufig. Kant's SW: Philosophen. Kant's

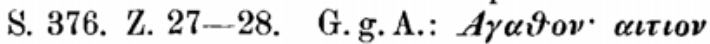

S. 376. Z. 28. G.g. A.: Plato

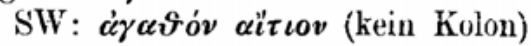

SW: Platon

S. 377. Z. 2. G.g. A.: Ausdruck

SW: Ausdrucke

S. 377. Z. 4. G. g. A.: Platonischen System

S. 377. Z. 12 . G.g. A.: Plato

SW: platonischen Systeme

sW: Platon

Zu Anhang 3: Replik Herbarts in der Neuen Leipziger Literatur-Zeitung (LLZ).

S. 388. Z. 6. I.IZ: Jenaer A. L.Z.

SW: jenaer allg. Lit. Zeitung

S. 388. Z. 21. LIZ: insbesondre

SW: insbesondere

S. 389. Z. 26. LLL: in deren Iösung

SW: in dieser Lösung

S. 390. Z. 5. LLZ: Heracl. $\gamma \varepsilon v$. in ovo. $P$.

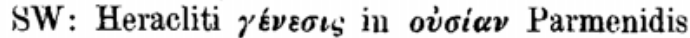

S. 390. Z. 5. LLZ: divide Her.

SW: divide Heracliti

S. 390. Z. 17. LLZ: (des Seyn)

SW: (das Seyn)

S. 391. Z. 26. LLZ: zu offenbarenden (s. Textveränder. S. Lxxıx)

SW : zu offenbarendem

S. 391. Z. 34. LLZ: einem Urbegriff

SW: einem Unbegriff

S. 391. Z. 41. LLZ: $\delta \delta \varepsilon$

SW: ¿̀ $\delta \dot{\eta}$

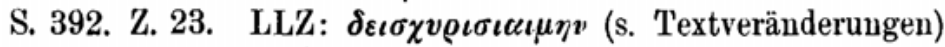

SW : $\delta \varepsilon \iota \sigma \chi v \varrho \iota \sigma \alpha i \mu \eta \nu$ 
S. 392. Z. 32. LLL: letztre

SW: letztere

S. 392. 'Z. 40. LLZ: angezogen

SW: ausgezogen

S. 393. Z. 18 . LLZ: vollkommner

SW: vollkommen

S. 393. Z. 38. LLZ: und der Vernunft

SW: und Vernunft

S. 394. Z. 11. LLZ: stehn

SW: stehen

S. 394. Z. 28. LLZ: Kategorieentafeln

SW: Kategorientafeln.

Paginirung. Im Texte der Abhandlung: De Platonici etc. ist die Paginirung des Originals angegeben, unter dem Texte diejenige der Nachdrucke in SW XII, 63-88 und $\mathrm{KLS} \mathrm{SCH}_{\mathrm{C}}{ }^{*} 69-97$.

Unter dem Texte der "Selbstanzeige“ steht die Paginirung des Nachdruckes in SW XII, XI-xII. Im Texte der Böckhschen „Recension" steht die Angabe der ,Spalten" des Originaldruckes. Im Texte der „Replik“ steht die Paginirung des Originaldruckes, unter dem Texte diejenige des Nachdruckes in SW XII, 88-96.

\section{Supplemente.}

I.

\section{Etwas über die allgemeinsten Ursachen, welche in den Staaten den Wachsthum und den Verfall der Moralität bewirken.}

Herbarts Glückwunschrede an die Abiturienten des Gymnasiums von Oldenburg, gehalten Ustern 1793. S. $397-407$.

In dem Osterprogramme (1793) des Gymıasiums zu Oldenburg berichtet der Rector J. S. Manso, nachdem er die Namen der valedicierenden Abiturienten genannt, folgendermassen: „Ihnen wird Johann Friedrich Herbart, aus Oldenburg, antworten, zu ihrem Vorhaben Glück wünschen, und dabey die allgemeinen Ursachen auseinandersetzen, welche in Staaten das Wachsthum und den Verfall der Moralität bewirken."

Da Manso im Programm von 1794 (S. 35) ausdrücklich erklärt, dass er ,weder an der Wahl der Naterien noch an der Ausführung einigen Antheil habe“, dass vielmehr alles was ,gut und nicht gut sein möchte in diesen Reden auf Rechnung der Redner komme", so ist anzunehmen, dass die Rede von Herbart ganz selbständig abgefasst worden ist. 
Die Rede erregte grossen Beifall und dieser war die Ursache, dass die Herausgeber der „Blätter vermischten Inhalts“, zu welchen auch G. A. von Halem, der spätere väterliche Freund Herbarts, gehörte, beschlossen, sie in dem genamnten Organe zu publiciren. Merkwürdigerweise erschien sie daselbst erst im 6. Bande, 1797. Welches die Gründe dieses späten Druckes gewesen sind, habe ich nicht in Erfahrung bringen können. Sie ist die früheste von Herbarts im Drucke erschienenen Schriften, zugleich aber auch überhaupt die einzige, welche aus Herbarts Schülerzeit noch rollständig erhalten ist.

Dracke. Es existirt nur der eine Abdruck in den „Blättern vermischten Inhalts" Bd. VI, S. 60-79.

Grundlage. Der 'Text in den „Blättern vermischten Inhalts.“

Textveränderungen.

S. 401. Z. 39. Wenn der Gärtner . . . statt . . Wen der Gärtner.

S. 404. Z. 36. auch den Tyrannen ... statt . . . auf den Tyrannen.

Paginirung. Im Texte ist die Paginirung des Originaltextes angegeben worden.

\section{II.}

1) Hartensteins Mittheilungen über Herbarts Aufsatz: „Etwas über die Lehre von der menschlichen Freiheit" [1790].

2) Herbarts Selbstkritik über seinen Aufsatz: „Etwas über die Lehre etc." [1800]. S. 409-413.

Da der Aufsatz: Etwas über die Lehre von der menschlichen Freiheit, welchen Herbart in seinem 14. Lebensjahre niederschrieb, nicht mehr erhalten zu sein scheint, denn trotz aller Recherchen ist es mir nicht gelungen seiner habhaft zu werden, so gebe ich das Fragment desselben, wie es in den Mittheilungen von Hartenstein ( $\mathrm{KLSCH} I, \mathrm{Ix})$ vorliegt.

Als Errgänzung dieses Aufsatzes und zugleich ais ein Dokument von Herbarts Entwickelungsstufe, die er 10 Jahre nach Abfassung des genannten Aufsatzes einnahm, dient die Selbstkritik über denselben Aufsatz. Auch das Manuscript zu diesem Aufsatze konnte nicht mehr aufgefunden werden. Es ist daher der von Hartenstein in $\mathrm{KL}_{\mathrm{L}} \mathrm{S} \mathrm{CH}$ I, S. $\mathbf{x}-\mathbf{x}$ II überlieferte Text, welcher die einzige Ueberlieferung ist, nachgedruckt worden.

\section{III.}

Joh. Rists Aufsatz: Ueber moralische und ästhetische Ideale. 1796. S. $415-420$.

Dieser kleine von Rist der literarischen Gesellschaft in Jena eingesandte Aufsatz, den Gries kritisirte, ist deshalb wichtig, weil er das 
Verständniss von Herbarts Aufsatz über den Begriff des Ideals (S. 5-8 vorl. Ausg.) wesentlich erleichtert. In dem genannten Aufsatze nahm Herbart seinen Freund Rist gegen die Angriffe von Gries in Schutz.

Das Manuscript zu dem obigen Aufsatze von Rist ist in dem Rist'schen Nachlasse nicht mehr aufzufinden. Herr G. Poel, der Herausgeber von Rists Autobiographie, dem ich die übrigen im Rist'schen Nachlasse noch vorhandenen Herbartiana verdanke, konnte über den Verbleib des Manuscriptes keine Auskunft ertheilen.

Drucke. Der Aufsatz ist nur einmal abgedruckt und zwar im Anhange von R. Zimmermanus Abhandlung über die „Perioden in Herbart's philosophischem Geistesgang" (Sitzungsberichte der (Wiener) Akademie der Wissenschaften, philos.-histor. Classe, Bd. 83, Jahrg. 1876, S. $227-231$.

Grundlage. Dieser von Zimmermann überlieferte Abdruck bat als Grundlage des Textes vorliegender Ausgabe gedient.

Paginirung. Im Texte ist die entsprechende Paginirung der „Sitzungsberichte etc." angegeben.

\section{IV.}

1) Herbarts Meldeschreiben zur Promotion und Habilitation.

2) Herbarts curriculum vitae. S. 421-424.

Beide Schriftstücke, von denen das curriculum das wichtigere ist, sind bis jetzt noch nicht veröffentlicht. Der philosophischen Facultät in Göttingen, welche mir die auf Herbart bezüglichen Akten zur Verfügung stellte, verdanke ich ihre Mittheilung. Herr Dr. Ehrenfeuchter, Custos an der Göttinger Universitätsbibliothek, war, da ich zur Zeit an einer Reise nach Göttingen verhindert war, so gütig, die obigen Schriftstücke für mich auszuziehen.

Leipzig, September 1881. 